\title{
INTEGRAL MODELS FOR MODULI SPACES OF $G$-TORSORS
}

\author{
MARTIN OLSSON
}

\section{INTRODUCTION}

The work in this paper is a generalizations to higher dimensions of a particular application of the Abramovich-Vistoli theory of twisted stable maps [AV] (and its 'tame version' of Abramovich-Olsson-Vistoli [AVO2]).

Let us begin by reviewing how the Abramovich-Vistoli theory gives compactifications of moduli spaces for curves with (possibly non-abelian) level structure.

1.1. Let $C / S$ be a smooth proper curve of genus $g$ over a scheme $S$, and let $P$ be a finite set of prime numbers which includes all residue characteristics of $S$. For any section $s: S \rightarrow C$ we then obtain, as in [DM, 5.5], a pro-object $\pi_{1}(X / S, s)$ in the category of locally constant sheaves of finite groups on $S$ whose fiber over a geometric $\bar{t} \rightarrow S$ is equal to the maximal prime to $P$ quotient of $\pi_{1}\left(C_{\bar{t}}, s_{\bar{t}}\right)$.

Now let $G$ be a finite group of order not in $P$. Let $\mathscr{H}_{o m^{\text {ext }}}\left(\pi_{1}(X / S, s), G\right)$ denote the sheaf of homomorphisms $\pi_{1}(X / S, s) \rightarrow G$ modulo the action of $\pi_{1}(X / S, s)$ given by conjugation. Then the sheaf $\mathscr{H} \mathrm{om}^{\text {ext }}\left(\pi_{1}(X / S, s), G\right)$ is a locally constant sheaf on $S$ which is canonically independent of the section $s$. It follows that for any smooth proper curve $C / S$ of genus $g$ there is a canonically defined sheaf $\mathscr{H}$ om $^{\text {ext }}\left(\pi_{1}(X / S), G\right)$ even when $C / S$ does not admit a section. Following [DM, 5.6], we define a Teichmüller structure of level $G$ on $C / S$ to be a section of $\mathscr{H} \mathrm{om}^{\text {ext }}\left(\pi_{1}(X / S), G\right)$, which étale locally on $S$ can be represented by a surjective homomorphism $\pi_{1}(X / S, s) \rightarrow G$ for a suitable section $s$. As in [DM, 5.8] we define ${ }_{G} \mathscr{M}_{g}$ to be the stack over $\mathbb{Z}[1 /|G|]$ which to any $\mathbb{Z}[1 /|G|]$-scheme $S$ associates the groupoid of pairs $(C / S, \sigma)$, where $C / S$ is a smooth proper genus $g$ curve and $\sigma$ is a Teichmüller structure of level $G$.

1.2. The space ${ }_{G} \mathscr{M}_{g}$ is connected with the Abramovich-Vistoli theory as follows. Let $C / S$ be a curve as above, and fix a section $s: S \rightarrow C$. Let ${ }_{G} \mathscr{K}_{g}^{\circ}$ denote the stack over $\mathbb{Z}[1 /|G|]$ which to any $\mathbb{Z}[1 /|G|]$-scheme $S$ associates the groupoid of principal $G$-bundles $P \rightarrow C$, such that for every geometric point $\bar{t} \rightarrow S$ the fiber $P_{\bar{t}} \rightarrow C_{\bar{t}}$ is connected. The choice of the section $s$ enables us to describe the stack ${ }_{G} \mathscr{K}_{g}^{\circ}$ as follows. For any object $P \rightarrow C$ of ${ }_{G} \mathscr{K}_{g}^{\circ}$, the pullback $s^{*} P$ is a $G$-torsor with action of $\pi_{1}(X / S, s)$ on $S$. Étale locally on $S$ we can choose a trivialization $\tilde{s}: S \rightarrow s^{*} P$ of the $G$-torsor $s^{*} P$, and such a trivialization defines a homomorphism

$$
\pi_{1}(X / S, s) \rightarrow G
$$

The assumption on the connectedness of the geometric fibers $P_{\bar{t}}$ implies that this map is surjective. It follows that the conjugacy class of the homomorphism $\pi_{1}(X / S, s) \rightarrow G$ is independent of the choice of $\tilde{s}$ and also independent of the section $s$. In this way we obtain 
a morphism

$$
{ }_{G} \mathscr{K}_{g}^{\circ} \rightarrow{ }_{G} \mathscr{M}_{g}
$$

This is in fact a morphism of stacks over $\mathscr{M}_{g}$ (the moduli stack classifying genus $g$ curves), and a straightforward verification, which we leave to the reader, shows that this maps identifies ${ }_{G} \mathscr{M}_{g}$ with the relative coarse moduli space, in the sense of $[\mathrm{AVO} 2, \S 3]$, of ${ }_{G} \mathscr{K}_{g}^{\circ} \rightarrow \mathscr{M}_{g}$.

On the other hand, the category of $G$-torsors over a smooth genus $g$-curve $C / S$ is equivalent to the category of morphisms $C \rightarrow B G$. Therefore the theory of twisted stable maps developed in $[\mathrm{AV}]$ gives a natural compactification ${ }_{G} \mathscr{K}_{g}$ of ${ }_{G} \mathscr{K}_{g}^{\circ}$ over $\mathbb{Z}[1 /|G|]$. Forgetting the $G$-torsor defines a morphism ${ }_{G} \mathscr{K}_{g} \rightarrow \overline{\mathscr{M}}_{g}$ extending 1.2.1, and therefore by passing to the associated relative coarse moduli space over $\overline{\mathscr{M}}_{g}$ we obtain a compactification of ${ }_{G} \mathscr{M}_{g}$.

1.3. It is natural to ask for an extension of the stable map spaces ${ }_{G} \mathscr{K}_{g}$ to schemes where $|G|$ is not invertible. This appears intractable in general, but as explained in [AVO2] if $G$ is a tame group scheme, as defined in loc. cit., then one can indeed develop a theory of twisted stable maps and the resulting moduli spaces ${ }_{G} \mathscr{K}_{g}$ are proper.

An interesting case to consider here is the case of $G=\mu_{n}$ for some integer $n$. This group scheme is tame, and so we obtain a proper moduli space $\mu_{n} \mathscr{K}_{g}$ over $\mathbb{Z}$ classifying $\mu_{n^{-}}$ torsors over twisted curves. The substack $\mu_{n} \mathscr{K}_{g}^{\circ} \subset \mu_{n} \mathscr{K}_{g}$ classifies smooth curves $C / S$ with a $\mu_{n}$-torsor $P \rightarrow C$. Giving such a torsor $P$ is in turn equivalent to giving a pair $(L, \iota)$, where $L$ is a line bundle on $C$ and $\iota: L^{\otimes n} \rightarrow \mathscr{O}_{C}$ is an isomorphism. As before there is a projection $\mu_{n} \mathscr{K}_{g}^{\circ} \rightarrow \mathscr{M}_{g}$ and since the automorphism group of any pair $(L, \iota)$ as above is canonically isomorphic to $\mu_{n}$ acting on $L$, it follows that the relative coarse moduli space of $\mu_{n} \mathscr{K}_{g}^{\circ} \rightarrow \mathscr{M}_{g}$ is equal to the $n$-torsion subgroup scheme of the Jacobian of the universal curve over $\mathscr{M}_{g}$. Therefore the spaces $\mu_{n} \mathscr{K}_{g}$ enable one to obtain proper models over $\mathbb{Z}$ of the $n$-torsion subgroup of the universal Jacobian. In the case $g=1$, a discussion of the models obtained in this way and their relationship with the Katz-Mazur regular models for moduli spaces of elliptic curves with level structure is given in [AVO2, §6].

1.4. The aim of this paper is to generalize the spaces ${ }_{G} \mathscr{K}_{g}$ to moduli spaces for $G$-torsors on higher dimensional varieties. The setup is the following. We consider a flat proper morphism of schemes $f: X \rightarrow S$ (or more generally algebraic stacks) which is semi-stable in a suitable sense (see 7.1 for the precise assumptions). The semistability assumption implies in particular that $f$ extends canonically to a morphism of $\log$ schemes $\left(X, M_{X}\right) \rightarrow\left(S, M_{S}\right)$ for certain $\log$ structures $M_{X}$ and $M_{S}$ on $X$ and $S$ respectively. For a tame group scheme $G / S$ we then construct a proper $S$-stack ${ }_{G} \mathscr{K}_{X / S}$ whose restriction to the open subset $S^{\circ} \subset S$ where $X \rightarrow S$ is smooth is simply the moduli stack of $G$-torsors on $X^{\circ}:=X \times{ }_{S} S^{\circ}$.

In the case when $S=\overline{\mathscr{M}}_{g}$ and $X$ is the universal curve over $\overline{\mathscr{M}}_{g}$, we obtain the spaces of stable maps ${ }_{G} \mathscr{K}_{g}$.

In general, the object of ${ }_{G} \mathscr{K}_{X / S}$ at the boundary can be described as follows. The key observation is that to any simple extension of $\log$ structures $j: M_{S} \hookrightarrow N_{S}$ on $S$ (see 2.1 for the precise definition) there is an associated tame stack $\mathscr{X}_{j} \rightarrow X$. The stack ${ }_{G} \mathscr{K}_{X / S}$ is the stack which to any $S$-scheme $g: T \rightarrow S$ associated the groupoid of pairs

$$
\left(j: g^{*} M_{S} \hookrightarrow N_{T}, \quad P \rightarrow \mathscr{X}_{j}\right),
$$


where $j$ is a simple extension with associated tame stack $\mathscr{X}_{j} \rightarrow X \times_{S} T$, and $P \rightarrow \mathscr{X}_{j}$ is a $G$-torsor. Furthermore this data is required to satisfy a suitable stability condition (see section 7). Let us summarize the main properties here:

Theorem 1.5. (i) The stack ${ }_{G} \mathscr{K}_{X / S}$ is a proper algebraic stack over $S$ with finite diagonal.

(ii) The stack ${ }_{G} \mathscr{K}_{X / S}$ is tame.

Remark 1.6. If the connected component of the identity in the relative Picard functor $\underline{\mathrm{Pic}}_{X / S}$ of $X / S$ is smooth over $S$, then ${ }_{G} \mathscr{K}_{X / S}$ is flat over $S$ with local complete intersection fibers. This follows from the same argument used in [AVO2, §5].

Remark 1.7. The assumptions on $X \rightarrow S$ imply that the morphism $X \rightarrow S$ is cohomologically flat in dimension 0 and therefore $\underline{\mathrm{Pic}}_{X / S}$ is an algebraic space [Ar, 7.3].

1.8. Acknowledgements: The author is grateful to Dan Abramovich and Angelo Vistoli for helpful conversations. The author was partially supported by NSF grant DMS-0714086, NSF CAREER grant DMS-0748718, and an Alfred P. Sloan Research Fellowship.

1.9. Conventions: We assume the reader is familiar with the basic of logarithmic geometry in the sense of Fontaine and Illusie (see for example [Ka] or $[\mathrm{Og}]$ ). In this paper we only consider fine log structures, and therefore usually omit the adjective "fine".

\section{Stacks ASsociated to Simple EXtensions.}

2.1. In general if $Z$ is a scheme and $h: M \rightarrow N$ is a morphism of fine log structures on $Z$ we say that $h$ is logarithmically semistable if for every geometric point $\bar{z} \rightarrow Z$ there exists isomorphisms $\bar{M}_{\bar{z}} \simeq \mathbb{N}^{r}$ and $\bar{N}_{\bar{z}} \simeq \mathbb{N}^{r+s}$ such that the map $\bar{M}_{\bar{z}} \rightarrow \bar{N}_{\bar{z}}$ is given by

$$
e_{i} \mapsto\left\{\begin{array}{cl}
e_{i} & \text { if } i \neq r \\
e_{r}+e_{r+1}+\cdots+e_{r+s} & \text { if } i=r
\end{array}\right.
$$

where $e_{i}$ denotes the $i$-th standard generator of $\mathbb{N}^{r}$.

We say that $h$ is a simple morphism if for every geometric point $\bar{z} \rightarrow Z$ there exists isomorphisms $\bar{M}_{\bar{z}} \simeq \mathbb{N}^{r}$ and $\bar{N}_{\bar{z}} \simeq \mathbb{N}^{r}$ for some integer $r$ such that the morphism $\bar{M}_{\bar{z}} \rightarrow \bar{N}_{\bar{z}}$ is given by

$$
\mathbb{N}^{r} \rightarrow \mathbb{N}^{r}, \quad e_{i} \mapsto a_{i} \cdot e_{i}
$$

for some collection of natural numbers $a_{1}, \ldots, a_{r} \in \mathbb{N}$.

2.2. Let $f:\left(X, M_{X}\right) \rightarrow\left(S, M_{S}\right)$ be a $\log$ smooth morphism with underlying morphism $X \rightarrow S$ proper. Assume further that the map of log structures $f^{*} M_{S} \rightarrow M_{X}$ is logarithmically semistable. This implies that for every geometric point $\bar{x} \rightarrow X$, with image in the singular locus of the morphism $X \rightarrow S$, there exists a unique irreducible element in $\bar{M}_{S, f(\bar{x})}$ whose image in $\bar{M}_{X, \bar{x}}$ is not irreducible. In this way we obtain for every geometric point $\bar{s} \rightarrow S$ a set map

$$
\left.\left\{\text { singular points of } X_{\bar{s}}\right\} \rightarrow \text { irreducible elements in } \bar{M}_{S, \bar{s}}\right\} .
$$

The morphism $f:\left(X, M_{X}\right) \rightarrow\left(S, M_{S}\right)$ is special and essentially semistable if for every geometric point $\bar{s} \rightarrow S$ this map induces a bijection between the connected components of the singular locus of $X_{\bar{s}}$ and the set of irreducible elements in $\bar{M}_{S, \bar{s}}$. 
2.3. Let $f:\left(X, M_{X}\right) \rightarrow\left(S, M_{S}\right)$ be a log smooth proper special and essentially semistable morphism. For a simple morphism $j: M_{S} \hookrightarrow N_{S}$ of fine log structures on $S$ we define an algebraic stack $\mathcal{X}_{j}$ over $X$ as follows. For any $X$-scheme $g: Y \rightarrow X$ define $\mathcal{X}_{j}(Y)$ to be the groupoid of commutative diagrams of fine log structures on $Y$

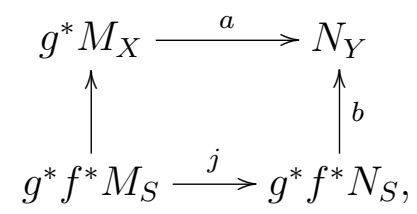

where $a$ is a simple morphism and $b$ is logarithmically semistable.

2.4. In local coordinates the stack $\mathcal{X}_{j}$ can be described as follows. First of all, as discussed in $[\mathrm{Ol}, 2.2]$ and $[\mathrm{MO}, 3.4]$ we can étale locally on $S$ and $X$ find a diagram of charts

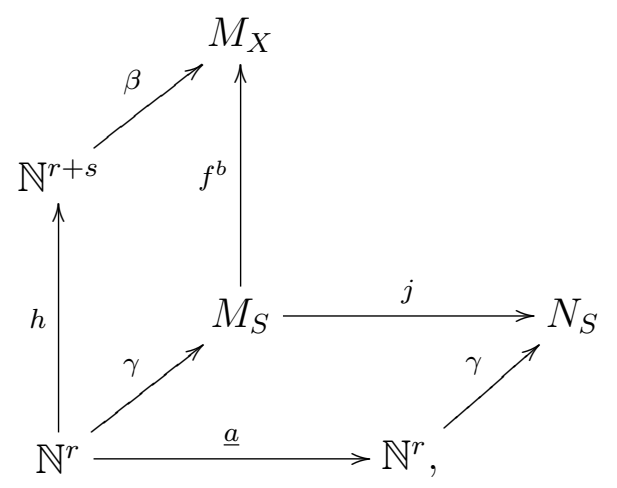

where $h$ is given by the formula 2.1 .1 and $\underline{a}$ denotes the map 2.1.2 for $\underline{a}=\left(a_{1}, \ldots, a_{r}\right)$. Let

$$
\chi_{\underline{a}}: \mathbb{N}^{r+s} \rightarrow \mathbb{N}^{r+s}
$$

denote the map given by

$$
e_{i} \mapsto a_{i} e_{i}, \quad i<r
$$

and

$$
e_{i} \mapsto a_{r} e_{i}, \quad i \geq r .
$$

We then have a commutative diagram

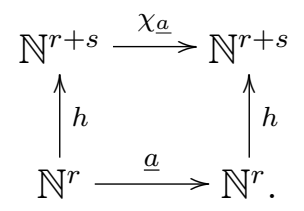

Let $\Delta$ denote the kernel of the map of group schemes

$$
\prod_{i=0}^{s} \mu_{a_{r}} \rightarrow \mu_{a_{r}}\left(\zeta_{0}, \ldots, \zeta_{s}\right) \mapsto \zeta_{0} \cdots \zeta_{s} .
$$

The commutative square 2.4.1 defines a morphism

$$
\delta: \mathbb{Z}\left[\mathbb{N}^{r}\right] \otimes_{\underline{a}, \mathbb{Z}\left[\mathbb{N}^{r}\right], h} \mathbb{Z}\left[\mathbb{N}^{r+s}\right] \rightarrow \mathbb{Z}\left[\mathbb{N}^{r+s}\right] .
$$

The group scheme $\Delta$ acts on $\operatorname{Spec}\left(\mathbb{Z}\left[\mathbb{N}^{r+s}\right]\right)$ over

$$
\operatorname{Spec}\left(\mathbb{Z}\left[\mathbb{N}^{r}\right] \otimes_{\underline{a}, \mathbb{Z}\left[\mathbb{N}^{r}\right], h} \mathbb{Z}\left[\mathbb{N}^{r+s}\right]\right) .
$$


If we write $\mathbb{Z}\left[z_{1}, \ldots, z_{r+s}\right]$ for $\mathbb{Z}\left[\mathbb{N}^{r+s}\right]$ then $\left(\zeta_{0}, \ldots, \zeta_{s}\right) \in \Delta$ acts by

$$
z_{i} \mapsto z_{i} \quad(i<r), \quad z_{i} \mapsto \zeta_{i-r} z_{i} \quad(i \geq r)
$$

Lemma 2.5. The stack $\mathcal{X}_{j}$ is isomorphic to the fiber product of the diagram

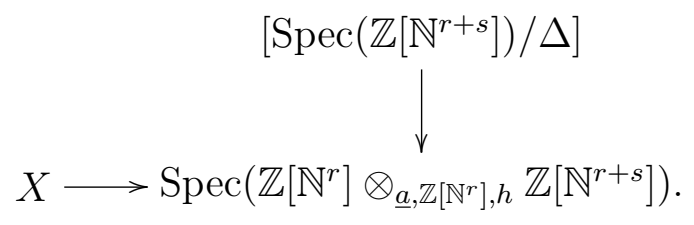

Proof. Let $\mathcal{S}$ denote the fiber product of the diagram 2.5.1. By [Ol4, 5.20] the stack $\mathcal{S}$ is isomorphic to the stack which to any scheme $g: Y \rightarrow X$ associates the groupoid of commutative diagrams of fine log structures

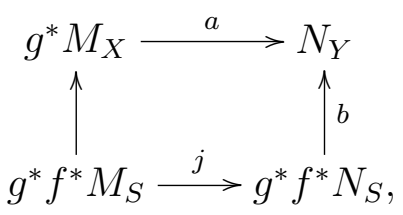

together with a morphism $\tau: \mathbb{N}^{r+s} \rightarrow \bar{N}_{Y}$ which locally on $Y$ lifts to a chart and such that the diagram

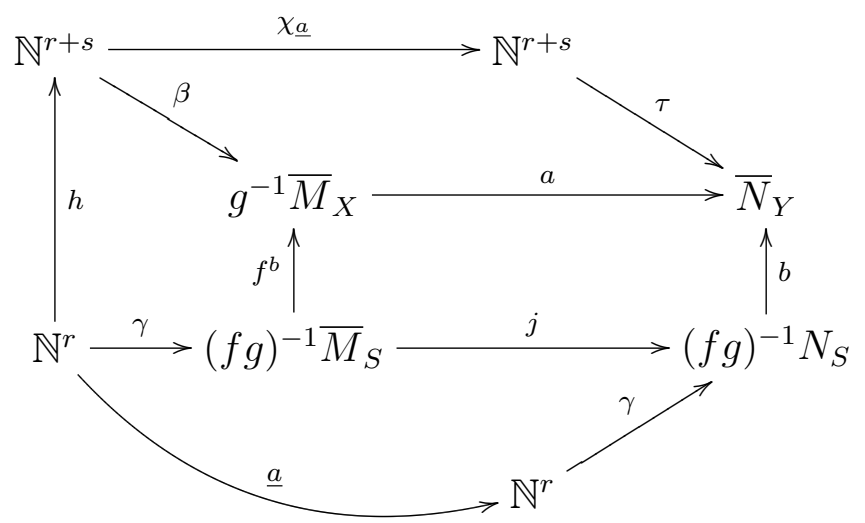

commutes. Note that the commutativity of 2.5.3 implies that the square 2.5.2 defines an object of $\mathscr{X}_{j}(Y)$. We therefore obtain a morphism of stacks

$$
\mathcal{S} \rightarrow \mathscr{X}_{j}
$$

over $X$. Furthermore, to prove that this is an equivalence it suffices to show that for any square 2.5.2 over $g: Y \rightarrow X$ defining an object of $\mathscr{X}_{j}(Y)$ there is a unique morphism $\tau: \mathbb{N}^{r+s} \rightarrow \bar{N}_{Y}$ which locally lifts to a chart and such that the diagram 2.5.3 commutes. By the definition of $\mathscr{X}_{j}$ for any geometric point $\bar{y} \rightarrow Y$ there exists such a map $\tau: \mathbb{N}^{r+s} \rightarrow \bar{N}_{Y, \bar{y}}$, and by spreading out we obtain the map $\tau$ in some étale neighborhood of $\bar{y}$. On the other hand, the map $\tau$ is clearly unique as the cokernel of $\chi_{\underline{a}}: \mathbb{N}^{r+s} \rightarrow \mathbb{N}^{r+s}$ is torsion and $\bar{N}_{Y}^{g p}$ is torsion free. Therefore these locally defined maps $\tau$ glue to a unique global map. 


\section{A DESCRIPTION OF G-TORSORS.}

It was shown in $[\mathrm{AVO}, 2.20]$ that if $G / S$ is a finite flat tame group scheme, then étale locally on $S$ the group $G$ can be written as an extension

$$
1 \rightarrow \Delta \rightarrow G \rightarrow H \rightarrow 1
$$

where $\Delta$ is a diagonalizable group scheme and $H$ is an étale group scheme of order invertible in $S$ (a tame étale group scheme). In what follows, when studying $G$-torsors it will often be convenient to reduce problems to the diagonalizable and tame étale cases separately. To this end, we discuss in this section some generalities about $G$-torsors for $G$ an extension as above.

3.1. Let $S$ be a scheme, and let $G / S$ be a finite flat group scheme which sits in an extension of group schemes

$$
1 \rightarrow \Delta \rightarrow G \rightarrow H \rightarrow 1
$$

where $\Delta$ is diagonalizable and $H$ is constant. Let $X$ be the character group of $\Delta$. For a $G$-torsor $P$ over $S$ let $\bar{P}$ denote the quotient $P / \Delta$, which is an $H$-torsor.

Since $\Delta$ is abelian the conjugation action of $G$ on $\Delta$ descends to an action

$$
\rho: H \rightarrow \operatorname{Aut}(\Delta) \text {. }
$$

For $h \in H$ the map $\rho_{h}: \Delta \rightarrow \Delta$ sends a local section $\delta \in \Delta$ to $\tilde{h} \delta \tilde{h}^{-1}$, where $\tilde{h} \in G$ is a (local) lifting of $h$.

3.2. For $h \in H$, let $Q_{h}$ denote the sheaf of liftings of $h$ to $G$. Then $Q_{h}$ is a $(\Delta, \Delta)$-bitorsor through the left and right translation actions.

For a $\Delta$-torsor $P$ let $Q_{h} \wedge P$ denote

$$
Q_{h} \wedge P:=Q_{h} \times P / \sim,
$$

where $\sim$ is the equivalence relation

$$
(q, p) \sim\left(q \delta^{-1}, \delta p\right), \quad q \in Q_{h}, \quad p \in P, \quad \delta \in \Delta
$$

The space $Q_{h} \wedge P$ is a $\Delta$-torsor with $\delta \in \Delta$ acting by

$$
\delta *(q, p)=(\delta q, p)
$$

Note that there is a canonical isomorphism of torsors

$$
Q_{h^{\prime}} \wedge Q_{h} \simeq Q_{h^{\prime} h},
$$

for $h, h^{\prime} \in H$.

3.3. Define $\mathscr{C}$ to be the category whose objects are collections of data

$$
\left(\bar{P}, P \rightarrow \bar{P},\left\{\chi_{h}\right\}_{h \in H}\right),
$$

as follows:

(1) $\bar{P}$ is an $H$-torsor over $S$.

(2) $P$ is a $\Delta$-torsor over $\bar{P}$.

(3) For each $h \in H$

$$
\chi_{h}: Q_{h} \wedge P \rightarrow h^{*} P
$$

is a morphism of $\Delta$-torsors over $\bar{P}$. 
(4) For any two elements $h, h^{\prime} \in H$ the diagram

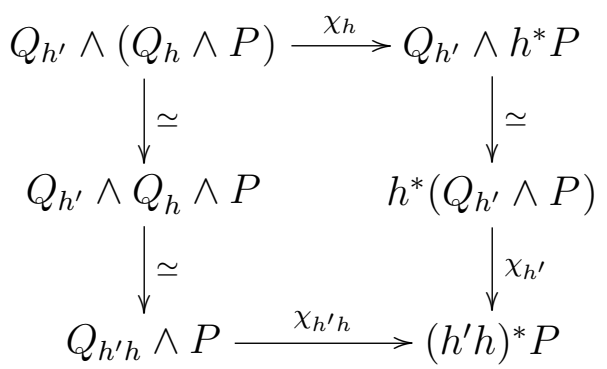

commutes.

3.4. There is a functor

$$
F:(G \text {-torsors over } S) \rightarrow \mathscr{C}
$$

defined as follows.

Given a $G$-torsor $P$ let $F(P)$ be the object of $\mathscr{C}$ given by $\bar{P}$ and the maps

$$
\chi_{h}: Q_{h} \wedge P \rightarrow h^{*} P
$$

given by the map

$$
Q_{h} \times P \rightarrow P
$$

provided by the action of $G$ on $P$. Then one verifies immediately that $\left(\bar{P}, P \rightarrow \bar{P},\left\{\chi_{h}\right\}\right)$ is an object of $\mathscr{C}$.

Proposition 3.5. The functor $F$ is an equivalence of categories.

Proof. Given an object $\left(\bar{P}, P \rightarrow \bar{P},\left\{\chi_{h}\right\}\right)$ the space $P$ inherits an action of $G$ by noting that

$$
G=\coprod_{h \in H} Q_{h}
$$

and therefore the maps

$$
\chi_{h}: Q_{h} \times P \rightarrow P
$$

define a map

$$
G \times P \rightarrow P .
$$

We leave to the reader the verification that this action makes $P$ a $G$-torsor, and that we obtain a quasi-inverse to $F$.

Proposition 3.6. Let $T$ be a regular scheme, and let $T^{\circ} \subset T$ be an open subset with $\operatorname{codim}\left(T \backslash T^{\circ}, T\right) \geq 2$. Let $G / T$ be a tame group scheme. Then the restriction functor

$$
(G \text {-torsors over } T) \rightarrow\left(G \text {-torsors over } T^{\circ}\right)
$$

is an equivalence of categories.

Proof. In the case when $G$ is an étale group scheme this follows from [SGA1, X.3.3].

In the case when $G$ is diagonalizable the result can be seen as follows. The scheme $G$ is isomorphic to a finite product of group schemes of the form $\mu_{n}$ so it suffices to consider the case when $G=\mu_{n}$. In this case the category of $G$-torsors is equivalent to the category of 
pairs $(L, \iota)$, where $L$ is a line bundle and $\iota$ is a trivialization of $L^{n}$. The result in this case therefore follows from the fact that the restriction functor

$$
\text { (line bundles on } T) \rightarrow\left(\text { line bundles on } T^{\circ}\right)
$$

is an equivalence of categories.

For the general case, we may work étale locally on $T$, and may therefore assume that there exists a short exact sequence

$$
1 \rightarrow \Delta \rightarrow G \rightarrow H \rightarrow 1
$$

where $\Delta$ is diagonalizable and $H$ is étale.

Let $\mathscr{C}\left(\right.$ resp. $\left.\mathscr{C}^{\circ}\right)$ be the category defined in 3.3 with $S=T\left(\operatorname{resp} . \quad S=T^{\circ}\right)$. Then it suffices to show that the restriction functor

$$
R: \mathscr{C} \rightarrow \mathscr{C}^{\circ}
$$

is an equivalence. For the essential surjectivity, let

$$
\left(\bar{P}^{\circ}, P^{\circ} \rightarrow \bar{P}^{\circ},\left\{\chi_{h}\right\}\right) \in \mathscr{C}^{\circ}
$$

be an object. By the case of an étale group scheme, $\bar{P}^{\circ}$ extends uniquely to an $H$-torsor $\bar{P} \rightarrow T$. Now by the case of a diagonalizable group scheme applied to $\bar{P}^{\circ} \subset \bar{P}$ the $\Delta$-torsor $P^{\circ} \rightarrow \bar{P}^{\circ}$ extends uniquely to a $\Delta$-torsor $P \rightarrow \bar{P}$, and furthermore the maps $\chi_{h}$ also extend uniquely. The resulting collection of data

$$
\left(\bar{P}, P \rightarrow \bar{P},\left\{\chi_{h}\right\}\right)
$$

defines an object of $\mathscr{C}$ as the commutativity of 3.3 .1 can be verified after restricting to $T^{\circ}$. This proves the essential surjectivity of $R$.

The full faithfulness is shown similarly.

For later use, let us also record the following mild generalization of 3.6:

Corollary 3.7. Let $\mathscr{X}$ be a regular algebraic stack over a base scheme $S$, and let $\mathscr{X}^{\circ} \subset \mathscr{X}$ be an open substack with complement of codimension $\geq 2$. Then for any tame group scheme $G$ over $S$ the restriction functor

$$
(G \text {-torsors over } \mathscr{X}) \rightarrow\left(G \text {-torsors over } \mathscr{X}^{\circ}\right)
$$

is an equivalence of categories.

Proof. Let $T \rightarrow \mathscr{X}$ be a smooth surjection, and let $T^{\circ} \subset \mathscr{X}$ be the inverse image of $\mathscr{X}^{\circ}$. By descent theory the category of $G$-torsors over $\mathscr{X}$ (resp. $\mathscr{X}^{\circ}$ ) is equivalent to the category of pairs $(P, \iota)$, where $P$ is a $G$-torsor over $T\left(\right.$ resp. $\left.T^{\circ}\right)$ and

$$
\operatorname{pr}_{1}^{*} P \rightarrow \operatorname{pr}_{2}^{*} P
$$

is an isomorphism of $G$-torsors over $T \times \mathscr{X} T\left(\right.$ resp. $\left.T^{\circ} \times \mathscr{X} \circ T^{\circ}\right)$ such that the usual cocycle condition on $T \times \mathscr{X} T \times \mathscr{X} T$ (resp. $T^{\circ} \times \mathscr{X} \circ T^{\circ} \times \mathscr{X}^{\circ} T^{\circ}$ ) holds. Therefore it suffices to show that for any $i \geq 1$ the restriction functor

$$
\left(G \text {-torsors over } T^{(i)}\right) \rightarrow\left(G \text {-torsors over } T^{(i) \circ}\right)
$$

is an equivalence of categories, where $T^{(i)}$ (resp. $T^{(i) \circ}$ ) denotes the $i$-fold fiber product of $T$ (resp. $T^{\circ}$ ) with itself over $\mathscr{X}\left(\right.$ resp. $\left.\mathscr{X}^{\circ}\right)$. This reduces the proof to the case when $\mathscr{X}$ is an 
algebraic space. In this case, repeating the above argument with an étale cover of $\mathscr{X}$ by a scheme we are then further reduced to the case of a scheme, which is 3.6.

\section{Extending torsors OVER Discrete VAluation RINGs.}

4.1. Let $A$ be a discrete valuation ring over $k$ with separably closed residue field $L$ and fraction field $K$. Fix a uniformizer $\pi \in A$.

Let $G / A$ be a tame finite flat group scheme. Let $\Delta$ be the connected component of the identity so we have an exact sequence

$$
1 \rightarrow \Delta \rightarrow G \rightarrow H \rightarrow 1
$$

where $H$ is tame and étale. Let $P_{\eta} \rightarrow \operatorname{Spec}(K)$ be a $G$-torsor.

For a positive integer $r$, let $A_{r}$ denote the finite $A$-algebra

$$
A[t] /\left(t^{r}-\pi\right)
$$

Let $K_{r}$ denote the field of fractions of $A_{r}$.

Proposition 4.2. There exists a unique integer $e=e\left(P_{\eta}\right)$ such that $\left.P_{\eta}\right|_{K_{e}}$ extends to $A_{e}$, and such that if $f$ is any other positive integer for which $\left.P_{\eta}\right|_{K_{f}}$ extends to $A_{f}$ then e|f.

Moreover, for any integer $f$ divisible by e an extension of $\left.P_{\eta}\right|_{K_{f}}$ to $\operatorname{Spec}\left(A_{f}\right)$ is unique up to unique isomorphism.

Proof. Let us first make some elementary observations about $\mu_{n}$-torsors over a scheme $S$. Consider the short exact sequence of fppf-sheaves

$$
0 \longrightarrow \mu_{n} \longrightarrow \mathbb{G}_{m} \stackrel{u \mapsto u^{n}}{\longrightarrow} \mathbb{G}_{m} \longrightarrow 0
$$

which gives rise to a long exact sequence

$$
0 \longrightarrow \mu_{n}(S) \longrightarrow \Gamma\left(S, \mathscr{O}_{S}^{*}\right) \stackrel{u \mapsto u^{n}}{\longrightarrow} \Gamma\left(S, \mathscr{O}_{S}^{*}\right) \longrightarrow H^{1}\left(S, \mu_{n}\right) \longrightarrow \operatorname{Pic}(S) .
$$

If $\operatorname{Pic}(S)=0$, which we assume for the rest of the proof, then this sequence identifies the isomorphism classes of $\mu_{n}$-torsors on $S$ with

$$
\Gamma\left(S, \mathscr{O}_{S}^{*}\right) / \Gamma\left(S, \mathscr{O}_{S}^{*}\right)^{n}
$$

If $P \rightarrow S$ is a $\mu_{n}$-torsor, then the corresponding class $[P] \in \Gamma\left(S, \mathscr{O}_{S}^{*}\right) / \Gamma\left(S, \mathscr{O}_{S}^{*}\right)^{n}$ can be described as follows. The $\mu_{n}$-torsor $P$ corresponds to a pair $(L, \iota)$, where $L$ is a line bundle on $S$ and $\iota: L^{\otimes n} \rightarrow \mathscr{O}_{S}$ is an isomorphism. Now since $\operatorname{Pic}(S)$ is trivial, the line bundle $L$ is trivial. Let $f \in L(S)$ be a basis. Then the image of $f^{\otimes n}$ under $\iota$ is an element $u \in \Gamma\left(S, \mathscr{O}_{S}^{*}\right)$ and we define $[P]$ to be the class of $u$. Note that a different choice of basis $f$ changes $u$ by an element of $\Gamma\left(S, \mathscr{O}_{S}^{*}\right)^{n}$ so the class $[P]$ is independent of this choice.

We apply this observation with $S$ either the spectrum of $A$ or $K$. Let $\nu: K^{*} \rightarrow \mathbb{Z}$ be the valuation, normalized so that $\nu(\pi)=1$. From the snake lemma applied to the commutative 
diagram

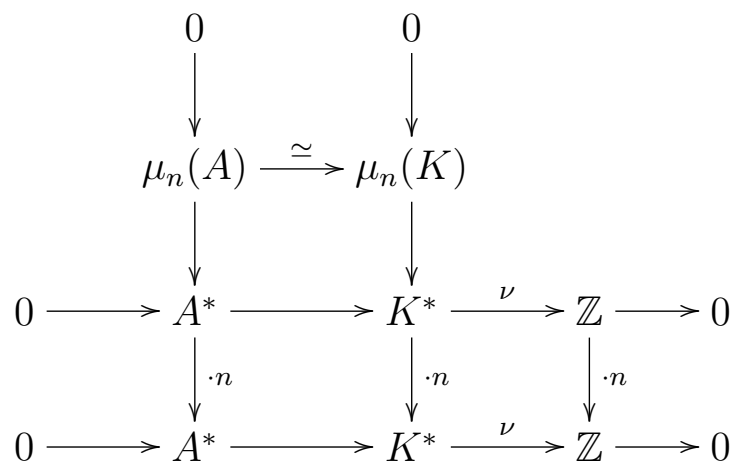

we obtain an exact sequence

$$
0 \longrightarrow A^{*} /\left(A^{*}\right)^{n} \longrightarrow K^{*} /\left(K^{*}\right)^{n} \stackrel{\nu}{\longrightarrow} \mathbb{Z} /(n) \longrightarrow 0 .
$$

If $P_{\eta} \rightarrow \operatorname{Spec}(K)$ is a $\mu_{n}$-torsor over $K$, then it follows that $P_{\eta}$ extends to a $\mu_{n}$-torsor over $A$ if and only if $\nu\left(\left[P_{\eta}\right]\right)=0$.

These remarks enable us to prove the proposition in the case when $G=\Delta$ is a diagonalizable group scheme as follows. If the Cartier dual of $\Delta$ is equal to $\mathbb{Z} /\left(n_{1}\right) \times \cdots \times \mathbb{Z} /\left(n_{r}\right)$, then the torsor $P_{\eta}$ corresponds to a collection of pairs $\left\{\left(L_{i}, \iota_{i}\right)\right\}_{i=1}^{r}$ where $L_{i}$ is a line bundle on $\operatorname{Spec}(K)$ and $\iota_{i}: L_{i}^{\otimes n_{i}} \rightarrow K$ is an isomorphism. Fix trivializations $s_{i} \in L_{i}$ and let $u_{i} \in K^{*}$ be the image of $s_{i}^{\otimes n}$ under $\iota_{i}$. Then it follows from the preceding discussion that $\left.P_{\eta}\right|_{K_{f}}$ extends to $A_{f}$ if and only if

$$
\nu\left(u_{i}\right) \cdot f \equiv 0 \quad\left(\bmod n_{i}\right)
$$

for all $i$. In this case we therefore take $e$ to be the least common multiple of the integers $n_{i} / \operatorname{gcd}\left(n_{i}, \nu\left(u_{i}\right)\right)$.

Note also that it is immediate that the functor

$$
(\Delta \text {-torsors over } \operatorname{Spec}(A)) \rightarrow(\Delta \text {-torsors over } \operatorname{Spec}(K))
$$

is fully faithful.

Next consider the case when $G=H$ is a tame étale group scheme. Fix an algebraic closure $K \subset \bar{K}$ and let $\pi_{1}(K)^{\prime}$ be the prime-to- $p$ fundamental group of $K$ with respect to this base point (where $p$ is the characteristic of the residue field $L$ ). Then since $L$ is separably closed we have a canonical isomorphism $\pi_{1}(K)^{\prime} \simeq \widehat{\mathbb{Z}}(1)^{\prime}$, where $\widehat{\mathbb{Z}}(1)^{\prime}$ denotes

$$
\widehat{\mathbb{Z}}(1)^{\prime}:=\lim _{n, p \nmid n} \mu_{n}(\bar{K}) \text {. }
$$

The torsor $P_{\eta}$ is then defined by a conjugacy class of homomorphisms

$$
\rho_{P_{\eta}}: \widehat{\mathbb{Z}}(1)^{\prime} \rightarrow H
$$

and $P_{\eta}$ extends to $\operatorname{Spec}(A)$ if and only if $\rho_{P_{\eta}}$ is trivial.

For any integer $f$, the restriction of $P_{\eta}$ to $K_{f}$ corresponds to the conjugacy class of homomorphisms defined by

$$
\pi_{1}\left(K_{f}\right)^{\prime} \simeq \widehat{\mathbb{Z}}(1)^{\prime} \stackrel{\cdot f}{\longrightarrow} \widehat{\mathbb{Z}}(1)^{\prime} \stackrel{\rho_{P_{\eta}}}{\longrightarrow} H
$$


It follows that in the case when $G$ is tame and étale we can take $e$ in the proposition to be the order of the image of $\rho_{P_{\eta}}$ (which depends only on the conjugacy class of $\rho_{P_{\eta}}$ ).

For the general case we combined the previous two cases as follows. Let $\bar{P}_{\eta}$ be the $H$-torsor $P_{\eta} / \Delta$ over $\operatorname{Spec}(K)$. Let $\bar{e}$ denote $e\left(\bar{P}_{\eta}\right)$, so we have an extension $\bar{P} \rightarrow \operatorname{Spec}\left(A_{\bar{e}}\right)$. Since the residue field of $A_{\bar{e}}$ is separably closed the torsor $\bar{P}$ is trivial, and therefore the underlying scheme of $\bar{P}$ is non-canonically isomorphic to

$$
\coprod_{h \in H} \operatorname{Spec}\left(A_{\bar{e}}\right)
$$

We can therefore apply the diagonalizable case to the $\Delta$-torsor $P_{\eta} \rightarrow \bar{P}_{\eta}$ for each connected component of $\bar{P}$. Let $e^{\prime}$ be the least common multiple of these integers and let $e=e^{\prime} \cdot \bar{e}$. Then by construction we obtain a $\Delta$-torsor $\left.P \rightarrow \bar{P}\right|_{A_{e}}$ restricting to $\left.P_{\eta}\right|_{K_{e}}$. Moreover, by the uniqueness of the extension in the diagonalizable case we obtain maps

$$
\chi_{h}: Q_{h} \wedge P \rightarrow h^{*} P
$$

of $\Delta$-torsors over $\bar{P}_{A_{e}}$ as in 3.3, inducing the given maps over $K_{e}$. These maps give $P$ the structure of a $G$-torsor extending $\left.P_{\eta}\right|_{K_{e}}$.

By a similar reasoning one obtains the uniqueness of the extension of $P_{\eta}$ and the remaining statements in the proposition.

Corollary 4.3. Let $V$ be a discrete valuation ring, and let $Y / V$ be a smooth $V$-scheme. Let $G / V$ be a tame finite flat group scheme, and assume $P_{\eta} \rightarrow Y_{\eta}$ is a $G$-torsor over the generic fiber $Y_{\eta}$ of $Y$. Then after making a finite flat base change $V \rightarrow V^{\prime}$ there exists a unique extension $P \rightarrow Y$ of $P_{\eta}$ to a $G$-torsor over $Y$.

Proof. Let $\pi \in V$ be a uniformizer. For each geometric point $\bar{\eta} \rightarrow Y$ mapping to a generic point of the closed fiber, the ring $\mathscr{O}_{Y, \bar{\eta}}$ is a discrete valuation ring with uniformizer the image of $\pi$. Therefore by 4.2 after making a finite ramified base change $V \rightarrow V^{\prime}$ we may assume that $P_{\eta}$ extends to $\operatorname{Spec}\left(\mathscr{O}_{Y, \bar{\eta}}\right)$ for each geometric point of the closed fiber. By a standard limit argument, it follows that $P_{\eta}$ extends to an étale neighborhood of each geometric generic point of the closed fiber. By the uniqueness statement in 4.2 these extensions are unique up to unique isomorphism, and therefore we get by descent an open subset $U \subset Y$ containing the generic fiber $Y_{\eta}$ and each generic point of the closed fiber, such that $P_{\eta}$ extends to $U$. By 3.6 it follows that $P_{\eta}$ extends to all of $Y$.

4.4. Let $f$ be an integer divisible by $e$. Let $\mathscr{V}_{f}$ denote the stack-theoretic quotient of $A_{f}$ by the action of $\mu_{f}$ for which $\zeta \in \mu_{f}$ sends $t$ to $\zeta$. Then

$$
\mathscr{V}_{f} \times_{\operatorname{Spec}(A)} \operatorname{Spec}(K) \simeq\left[\operatorname{Spec}\left(K_{f}\right) / \mu_{f}\right] \simeq \operatorname{Spec}(K),
$$

and the resulting inclusion

$$
\operatorname{Spec}(K) \hookrightarrow \mathscr{V}_{f}
$$

is a dense open immersion.

The stack $\mathscr{V}_{f}$ has the following modular description: For any $A$-scheme $g: T \rightarrow \operatorname{Spec}(A)$ the category $\mathscr{V}_{f}(T)$ is equivalent to the category of triples $(M, \gamma, \iota)$, where $M$ is an invertible 
sheaf on $T, \gamma: M \rightarrow \mathscr{O}_{T}$ is a morphism of line bundles, and $\iota: M^{\otimes f} \rightarrow \mathscr{O}_{T}$ is an isomorphism of line bundles on $T$ such that the diagram

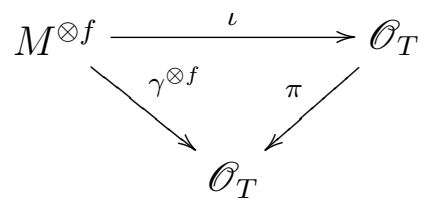

commutes.

In terms of this description, the inclusion $\operatorname{Spec}(K) \hookrightarrow \mathscr{V}_{f}$ corresponds to the trivial line bundle $L=K \cdot b$ with the map $\gamma$ sending $b$ to $1 \in K$ and the isomorphism $\iota$ being given by multiplication by $\pi^{-1}$.

Proposition 4.5. The torsor $P_{\eta} \rightarrow \operatorname{Spec}(K)$ extends uniquely to a $G$-torsor $\mathscr{P}_{f} \rightarrow \mathscr{V}_{f}$.

Proof. Let $P \rightarrow \operatorname{Spec}\left(A_{f}\right)$ denote the extension of $\left.P_{\eta}\right|_{K_{f}}$ provided by 4.2 . Let

$$
\rho: \operatorname{Spec}\left(A_{f}\right) \times \mu_{f} \rightarrow \operatorname{Spec}\left(A_{f}\right)
$$

be the map giving the action. To give an extension of $P$ to $\mathscr{V}_{f}$ is equivalent to giving an isomorphism

$$
\gamma: \operatorname{pr}_{1}^{*} P \rightarrow \rho^{*} P
$$

over $\operatorname{Spec}\left(A_{f}\right) \times \mu_{f}$ satisfying the following cocycle condition (thereby giving descent data for $P$ to $\left.\mathscr{V}_{f}\right)$. Let

$$
m: \mu_{f} \times \mu_{f} \rightarrow \mu_{f}
$$

be the map giving the group multiplication. Then the cocycle condition on $\gamma$ is that the diagram over $\operatorname{Spec}\left(A_{f}\right) \times \mu_{f} \times \mu_{f}$

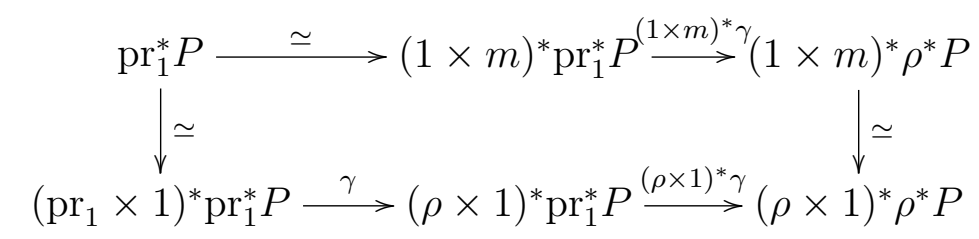

commutes. From this it follows that if an extension exists then it is unique up to unique isomorphism.

To construct the extension $\mathscr{P}_{f}$, consider first the case when $G=\mu_{n}$ for some integer $n$. In this case, the torsor $P_{\eta}$ corresponds to the trivial line bundle $L_{\eta}=K \cdot b$ with an isomorphism

$$
L_{\eta}^{\otimes n}=K \cdot b^{\otimes n} \rightarrow K
$$

sending $b^{\otimes n}$ to some element $g \in K^{*}$. After possibly changing our choice of basis $b$ for $L_{\eta}$ we may assume that $g \in A$. Furthermore, by definition of the integer $e$, if $r$ is the valuation of $g$ then the integer $s:=n / \operatorname{gcd}(n, r)$ divides $f$. Let $k$ denote $f / s$.

Let $\left(M, \gamma: M \rightarrow \mathscr{O}_{\mathscr{V}_{f}}, \iota: M^{\otimes f} \simeq \mathscr{O}_{\mathscr{V}_{f}}\right)$ be the tautological object over $\mathscr{V}_{f}$. Set

$$
N:=M^{k r / \operatorname{gcd}(n, r)},
$$

let $\gamma_{N}: N \rightarrow \mathscr{O}_{\mathscr{V}_{f}}$ denote the map

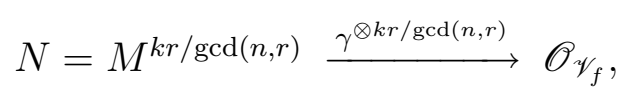


and let $\iota_{N}: N^{\otimes n} \rightarrow \mathscr{O}_{\mathscr{V}}$ be the isomorphism

$$
N^{\otimes n} \stackrel{\simeq}{\longrightarrow} M^{k r s} \stackrel{\simeq}{\longrightarrow}\left(M^{f}\right)^{r} \stackrel{\iota^{\otimes r}}{\longrightarrow} \mathscr{O}_{\mathscr{V}_{f}}
$$

We then have a commutative diagram

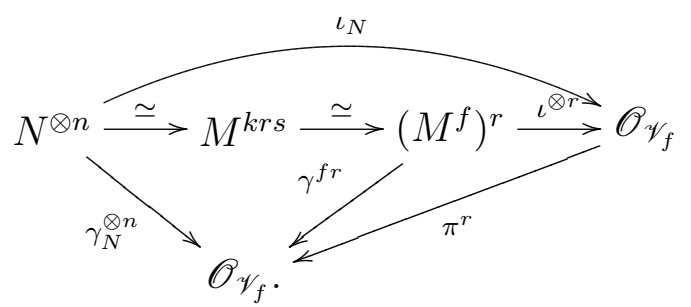

Let $L=A \cdot b$ denote the trivial line bundle on $\operatorname{Spec}(A)$ which comes equipped with a map $\beta: L^{\otimes n} \rightarrow A$ sending $b^{\otimes n}$ to $g \in A$. Over $\mathscr{V}_{f}$ we then get a line bundle $\left.L\right|_{\mathscr{V}_{f}} \otimes N^{-1}$ which comes equipped with an isomorphism

$$
\left.\left(\left.L\right|_{\mathscr{V}_{f}} \otimes N^{-1}\right)^{n} \stackrel{\simeq}{\longrightarrow} L^{\otimes n}\right|_{\mathscr{V}_{f}} \otimes N^{-n} \stackrel{\beta \otimes \gamma_{N}^{-n}}{\longrightarrow}\left(\mathscr{O}_{\mathscr{V}_{f}}\right) \cdot g \cdot \pi^{-r} \stackrel{\simeq}{\longrightarrow} \mathscr{O}_{\mathscr{V}_{f}}
$$

This defines the extension in the case of $G=\mu_{n}$.

From this we also obtain the extension in the case when $G$ is diagonalizable as in the proof of 4.2 .

In the case when $G=H$ is a tame étale group scheme, let $\mathscr{P} \rightarrow \mathscr{V}_{f}$ be the normalization of $\mathscr{V}_{f}$ in $P_{\eta}$. By the construction of the integer $e$, the stack $\mathscr{P}$ is an $H$-torsor over $\mathscr{V}_{f}$ as this can be verified after pulling back to $\operatorname{Spec}\left(A_{f}\right)$. In fact the pullback of $\mathscr{P}$ to $\operatorname{Spec}\left(A_{f}\right)$ is trivial (as $A_{f}$ is strictly henselian local) and therefore as a stack $\mathscr{P}$ is isomorphic to a finite disjoint union of the form

$$
\mathscr{P} \simeq \coprod_{j}\left[\operatorname{Spec}\left(A_{f}\right) / \mu_{f_{j}}\right]
$$

for some integers $f_{j} \mid f$, where the action of $\mu_{f_{j}}$ is through the natural inclusion $\mu_{f_{j}} \subset \mu_{f}$.

For the general case one proceeds as in the proof of 4.2 using the diagonalizable and tame étale cases by writing $G$ as an extension

$$
1 \rightarrow \Delta \rightarrow G \rightarrow H \rightarrow 1
$$

where $\Delta$ is diagonalizable and $H$ is tame and étale, and considering the quotient $H$-torsor $P_{\eta} / \Delta$. We leave the details to the reader.

4.6. There is a more global version of 4.5 which we now describe.

Let $X$ be a smooth scheme over a separably closed field, and let $D \subset X$ be a connected divisor with local normal crossings. Let $X^{\circ}$ denote the complement $X \backslash D$, and let $P^{\circ} \rightarrow X^{\circ}$ be a $G$-torsor.

The divisor $D$ defines a log structure $M_{D}$ on $X$. For a geometric point $\bar{x} \rightarrow X$ the stalk $\bar{M}_{D, \bar{x}}$ is equal to the free monoid on generators the branches of $D$ at $\bar{x}$. Let $\mathscr{X}_{D, r}$ be the stack over $X$ which to any $X$-scheme $f: T \rightarrow X$ associates the groupoid $\mathscr{X}_{D, r}(T)$ of morphisms of 
$\log$ structures $f^{*} M_{D} \rightarrow M_{T}$ on $T$ such that for every geometric point $\bar{t} \rightarrow T$ there exists a commutative diagram

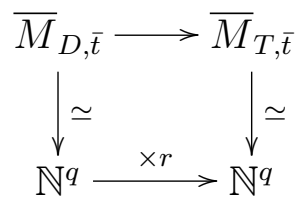

for some integer $q$.

Locally the stack $\mathscr{X}_{D, r}$ can be described as follows. Suppose given elements $f_{1}, \ldots, f_{q} \in \mathcal{O}_{X}$ such that $D$ is defined by $f_{1} \cdots f_{q}$ and the zero locus of each $f_{i}$ is a smooth divisor in $X$. Then $\mathscr{X}_{D, r}$ is the quotient of

$$
\operatorname{Spec}_{X}\left(\mathscr{O}_{X}\left[t_{1}, \ldots, t_{q}\right] /\left(t_{i}^{r}-f_{i}\right)\right)
$$

by the action of $\mu_{r}^{q}$ given by

$$
\left(\zeta_{1}, \ldots, \zeta_{q}\right) * t_{i}=\zeta_{i} \cdot t_{i}
$$

In particular, if $\bar{\eta} \rightarrow D$ is a geometric generic point and $A:=\mathscr{O}_{X, \bar{\eta}}$ (which is a discrete valuation ring with separably closed residue field) then we have

$$
\mathscr{V}_{r} \simeq \mathscr{X}_{D, r} \times_{X} \operatorname{Spec}(A)
$$

where $\mathscr{V}_{r}$ is defined as in 4.4 . Let $e_{\bar{\eta}}$ denote the least integer such that the $G$-torsor

$$
\operatorname{Spec}(A) \times_{X} P^{\circ} \rightarrow \operatorname{Spec}(A) \times_{X} X^{\circ}
$$

extends to $\mathscr{V}_{e_{\bar{\eta}}}$.

Also note that this local description implies that $\mathscr{X}_{D, r}$ is flat over $X$.

Proposition 4.7. The G-torsor $P^{\circ} \rightarrow X^{\circ}$ extends to $\mathscr{X}_{D, r}$ if and only if $e_{\bar{\eta}}$ divides $r$ for all geometric generic points $\bar{\eta} \rightarrow D$. In this case the extension is unique up to unique isomorphism.

Proof. If $P^{\circ}$ extends to $\mathscr{X}_{D, r}$ then $P^{\circ} \times_{X} \operatorname{Spec}(A)$ also extends to

$$
\mathscr{X}_{D, r} \times_{X} \operatorname{Spec}(A) \simeq \mathscr{V}_{r}
$$

and therefore by 4.5 we must have $e_{\bar{\eta}} \mid r$.

Conversely suppose $e_{\bar{\eta}} \mid r$ for all $\bar{\eta}$. Then $P^{\circ}$ extends to $\mathscr{X}_{D, r} \times_{X} \operatorname{Spec}(A)$, and by a standard limit argument there exists an étale neighborhood $U \rightarrow X$ of the generic points of $D$ such that $P^{\circ}$ extends to $\mathscr{X}_{D, r} \times_{X} U$. By the uniqueness if $V \subset X$ denotes the union of $X^{\circ}$ and the image of $U$ then $P^{\circ}$ extends to $V \times_{X} \mathscr{X}_{D, r}$.

Étale locally on $X$ we can write

$$
\mathscr{X}_{D, r} \simeq\left[\operatorname{Spec}(R) / \mu_{r}^{q}\right] .
$$

where $\operatorname{Spec}(R)$ is regular and finite over $X$. Now observe that the complement of the preimage of $V$ in $\operatorname{Spec}(R)$ has codimension $\geq 2$, and therefore the restriction of $P^{\circ}$ to $\operatorname{Spec}(R) \times_{X} X^{\circ}$ extends by 3.6 uniquely to a $G$-torsor $P \rightarrow \operatorname{Spec}(R)$. 
There is an extension of the $\mu_{r}^{q}$-action on $\operatorname{Spec}(R)$ to an action on $P$. Indeed suppose $X=\operatorname{Spec}(B)$, so that $R$ is a finite flat $B$-algebra. Let $\mathcal{O}_{P}$ denote the coordinate ring of $P$. The ring $\mathcal{O}_{P}$ is a finite flat $R$-algebra, and hence also a finite flat $B$-algebra. Let

$$
\tau: R \rightarrow R \otimes_{B} \mathcal{O}_{\mu_{r}^{q}}
$$

be the map defining the action of $\mu_{r}^{q}$ on $R$. Giving an extension of the $\mu_{r}^{q}$-action on $\operatorname{Spec}(R)$ to $P$ is equivalent to giving a coaction morphism

$$
\tilde{\tau}: \mathcal{O}_{P} \rightarrow \mathcal{O}_{P} \otimes_{R, \tau}\left(R \otimes_{B} \mathcal{O}_{\mu_{r}^{q}}\right) .
$$

Now both $\mathcal{O}_{P}$ and $\mathcal{O}_{P} \otimes_{R, \tau}\left(R \otimes_{B} \mathcal{O}_{\mu_{r}^{q}}\right)$ are projective $B$-modules, so the coaction map over $V$ extends uniquely to a morphism $\tilde{\tau}$. That this map $\tilde{\tau}$ satisfies the axioms for a coaction is immediate as these axioms can be verified after restricting to a dense open subset of $X$.

This action of $\mu_{r}^{q}$ on $P$ then gives descent data for $P$ to $\mathscr{X}_{D, r}$, completing the proof of the proposition.

\section{More extension Results.}

In this section we gather together some more local results about extending $G$-torsors. These results will be used in section 11 to verify the valuative criterion for properness for the stacks ${ }_{G} \mathscr{K}_{X / S}$.

Throughout this section $V$ denotes a discrete valuation ring with residue characteristic $p$ (possibly equal to 0 ).

Proposition 5.1. Let $n \geq 2$ be an integer, and let $X$ denote the spectrum of the strict henselization of

$$
V\left[x_{1}, \ldots, x_{n}\right] /\left(x_{1} \cdots x_{s}\right), \quad(s \leq n)
$$

at the point $\left\{x_{1}=\cdots x_{n}=0\right\}$. Let $X_{\text {sing }} \subset X$ denote the singular locus of $X$, and let $X^{\circ} \subset X$ denote the complement of the intersection of $X_{\text {sing }}$ with the closed fiber. Let $G / V$ be a tame group scheme. Then the restriction functor

$$
(G \text {-torsors on } X) \rightarrow\left(G \text {-torsors on } X^{\circ}\right)
$$

is an equivalence of categories.

The proof of 5.1 will be in several steps (5.2)-(5.15).

5.2. Let $\mathcal{R}$ denote the coordinate ring of $X$, and let $\mathfrak{m} \subset \mathcal{R}$ denote the maximal ideal. We begin the proof with some observations about the $\operatorname{ring} \mathcal{R}$. For a subset $I \subset\{1, \ldots, s\}$, let $\mathcal{R}_{I}$ denote the quotient of $\mathcal{R}$ by the ideal generated by $x_{i}(i \in I)$. More generally, for an $\mathcal{R}$-algebra $A$, let $A_{I}$ denote $A \otimes_{\mathcal{R}} \mathcal{R}_{I}$. Let $C_{A}$ denote the complex

$$
0 \rightarrow A \rightarrow \prod_{I,|I|=1} A_{I} \rightarrow \prod_{I,|I|=2} A_{I} \rightarrow \cdots
$$

where for $I \subset\{1, \ldots, s\}$ we write $|I|$ for the cardinality of $I$. The differentials in $C_{A}$ are obtained by taking alternating sums of the restriction maps. If $A=\mathcal{R}$ we write simply $C$. for $C_{\mathcal{R}}$, and if $A=\mathcal{R} / \mathfrak{m}^{t+1}$ we write $C_{t}$ for $C_{\mathcal{R} / \mathfrak{m}^{t+1}}$. Finally we write $\mathfrak{m}^{t} C_{A}$ for the image of the natural map of complexes

$$
\mathfrak{m}^{t} \otimes_{\mathcal{R}} C_{A}^{\cdot} \rightarrow C_{A}^{\cdot}
$$


Note that $\mathfrak{m}^{0} C_{A}=C_{A}$. As above we write simply $\mathfrak{m}^{t} C$ for $\mathfrak{m}^{t} C_{\mathcal{R}}$.

Lemma 5.3. For every $t \geq 0$, the complex $\mathfrak{m}^{t} C$ is acyclic.

Proof. Let $S=V\left[x_{1}, \ldots, x_{n}\right] /\left(x_{1} \cdots x_{s}\right)$, and for $I \subset\{1, \ldots, s\}$ let $S_{I}$ denote the quotient of $S$ by the ideal generated by $x_{i}(i \in I)$. Let $K$ denote the complex

$$
0 \rightarrow S \rightarrow \prod_{I,|I|=1} S_{I} \rightarrow \prod_{I,|I|=2} S_{I} \rightarrow \cdots
$$

defined analogously to $C$. Also let $J \subset S$ be the ideal generated by $\left(x_{1}, \ldots, x_{n}\right)$, and let $J^{t} K$ denote the image of the natural map of complexes

$$
J^{t} \otimes K \rightarrow K
$$

Then $J^{t} K$ defines a complex of coherent sheaves on $\operatorname{Spec}(S)$, whose stalk at the point defined by $x_{1}=x_{2}=\cdots=x_{n}=0$ is the complex $\mathfrak{m}^{t} C$. It therefore suffices to show that the complex $J^{t} K$ is acyclic.

For this note that there is a natural grading on $J^{t} K$ induced by the action of $\mathbb{G}_{m}^{n}$ given by

$$
\left(u_{1}, \ldots, u_{n}\right) * x_{i}=u_{i} x_{i}
$$

This grading breaks up $J^{t} K$ into the direct sum of complexes of the following form. Let $\left(a_{1}, \ldots, a_{n}\right) \in \mathbb{N}^{n}$ be such that $\sum a_{i} \geq t$, and let $\Sigma=\left\{i \in\{1, \ldots, s\} \mid a_{i} \neq 0\right\}$. Associated to this data is the complex

$$
k \rightarrow \prod_{|I|=1, I \cap \Sigma=\emptyset} k \rightarrow \prod_{|I|=2, I \cap \Sigma=\emptyset} k \rightarrow \cdots
$$

where again the transition maps are given by restriction. Since this complex is clearly acyclic, and $J^{t} K^{\cdot}$ is isomorphic to a direct sum of such complexes, it follows that $J^{t} K^{\cdot}$ is also acyclic.

Corollary 5.4. For every $t \geq 0$ the complex $C_{t}$ is acyclic.

Proof. For $t \geq 0$ let $\kappa_{t}$ denote the quotient complex

$$
\kappa_{t}:=\mathfrak{m}^{t} C^{\cdot} / \mathfrak{m}^{t+1} C^{.} .
$$

Since $\mathfrak{m}^{t} C$ and $\mathfrak{m}^{t+1} C$ are acyclic, the complex $\kappa_{t}$ is also acyclic. Furthermore, for every $t$ there is an exact sequence of complexes

$$
0 \rightarrow \kappa_{t} \rightarrow C_{t+1} \rightarrow C_{t} \rightarrow 0
$$

It therefore suffices to prove that the complex $C_{0}$ is acyclic. This is the complex

$$
0 \rightarrow k \rightarrow \prod_{I,|I|=1} k \rightarrow \prod_{I,|I|=2} k \rightarrow \cdots
$$

which is clearly acyclic.

5.5. We will also need a multiplicative version of the above results. For an $\mathcal{R}$-algebra $A$ we can also consider the following complex, which will be denoted $D_{A}$,

$$
A^{*} \rightarrow \prod_{I,|I|=1} A_{I}^{*} \rightarrow \prod_{I,|I|=2} A_{I}^{*} \rightarrow \cdots,
$$


where as before the transition maps are obtained from the natural restriction maps.

Fix now a flat $\mathcal{R}$-algebra $A$ which is equal to the strict henselization of a finite type $V$ algebra at some point, and let $A_{t}$ denote $A / \mathfrak{m}^{t+1} A$.

Lemma 5.6. For any $t \geq 0$ the complex $D_{A_{t}}$ is acyclic.

Proof. Let $J \subset\{1, \ldots, s\}$ denote the subset of indices $i$ for which $x_{i}$ maps to the maximal ideal of $A$. Then the complex $D_{A}^{*}$ is given by

$$
A^{*} \rightarrow \prod_{I \subset J,|I|=1} A_{I}^{*} \rightarrow \prod_{I \subset J,|I|=2} A_{I}^{*} \rightarrow \cdots
$$

and similarly for $D_{A_{t}}$.

We prove the lemma by induction on $t$. For $t=0$, note that the complex

$$
\prod_{I \subset J,|I|=1} A_{0}^{*} \rightarrow \prod_{I,|I|=2} A_{0}^{*} \rightarrow \cdots
$$

computes the cellular homology of the $|J|$-simplex with coefficients in $A_{0}^{*}$.

For general $t$, note that there is a natural exact sequence of complexes

$$
0 \rightarrow \kappa_{t} \otimes_{\mathcal{R}} A \rightarrow D_{A_{t}} \rightarrow D_{A_{t-1}} \rightarrow 0
$$

and as remarked above the complex $\kappa_{t}$ is acyclic.

Lemma 5.7. Let $\left\{M_{t}^{\cdot}\right\}_{t \geq 0}$ be a projective system of complexes of abelian groups such that for every $t$ and $i$ the map $M_{t}^{i} \rightarrow M_{t-1}^{i}$ is surjective. Let $M$ denote $\lim _{t} M_{t}$. If each $M_{t}$ is acyclic, then $M \cdot$ is also acyclic.

Proof. Let $x \in M^{i}$ be a closed element, and let $\left(x_{t}\right)_{t \geq 0}$ be its reductions. Suppose for some $t$ an element $y_{t} \in M_{t}^{i-1}$ has been chosen such that $d y_{t}=x_{t}$. We claim that then we can find an element $y_{t+1} \in M_{t}^{i-1}$ reducing to $y_{t}$ and such that $d y_{t+1}=x_{t+1}$. This clearly suffices to prove the lemma, for a compatible sequence of elements $\left(y_{t}\right)_{t \geq 0}$ with $d y_{t}=x_{t}$ for all $t$ defines an element $y \in M^{i-1}$ such that $d y=x$.

Let $H_{t}$ denote the kernel of the surjection

$$
M_{t+1} \rightarrow M_{t}
$$

so we have an exact sequence of complexes

$$
0 \rightarrow H_{t} \rightarrow M_{t+1} \rightarrow M_{t} \rightarrow 0
$$

This sequence implies in particular that $H_{t}$ is acyclic.

Now given $y_{t}$ as above, choose first any lifting $y_{t+1}^{\prime} \in M_{t+1}^{i-1}$ of $y_{t}$. Then $d y_{t+1}^{\prime}-x_{t+1}$ may not be zero, but this element is a closed element of $M_{t+1}^{i}$ mapping to zero in $M_{t}^{i}$. It therefore gives a closed element of $H_{t}^{i}$. Since $H_{t}$ is acyclic we can find an element $z \in H_{t}^{i-1}$ such that $d z=d y_{t+1}-x_{t+1}$. Letting $y_{t+1}:=y_{t+1}^{\prime}-z$ we obtain the desired lifting of $y_{t}$.

Corollary 5.8. Let $\widehat{A}$ denote the $\mathfrak{m}$-adic completion of $A$. Then the complex $D_{\widehat{A}}$ is acyclic.

Proof. This follows from 5.6 and 5.7, combined with the fact that $D_{\widehat{A}}^{\cdot}=\lim _{t} D_{A_{t}}$. 
Lemma 5.9. The complex $D_{A}$ is acyclic.

Proof. Let $x \in D_{A}^{i}$ be a closed element. Consider the functor

$$
F_{x}:(A \text {-algebras }) \rightarrow \text { Set },
$$

which to any $A$-algebra $B$ associates the set

$$
\left\{y \in D_{B}^{i-1} \mid d y=x\right\},
$$

where we abusively write also $x$ for its image in $D_{B}^{i}$. This functor is clearly locally of finite presentation in the sense of $[\operatorname{Ar} 2,1.5]$, and $F_{x}(\widehat{A})$ is non-empty by 5.8. By the Artin approximation theorem $[\operatorname{Ar} 2,1.12]$ it follows that $F_{x}(A)$ is also nonempty.

5.10. For $I \subset\{1, \ldots, s\}$, let $X_{I} \subset X$ denote $\operatorname{Spec}\left(\mathcal{R}_{I}\right) \subset \operatorname{Spec}(\mathcal{R})$. We write simply $\mathscr{O}_{X_{I}}$ and $\mathscr{O}_{X_{I}}^{*}$ for the pushforwards of these sheaves to $X$. The above results can be sheafified as follows. Again using the natural restriction maps we get complexes

$$
\mathscr{O}_{X} \rightarrow \prod_{I,|I|=1} \mathscr{O}_{X_{I}} \rightarrow \prod_{I,|I|=2} \mathscr{O}_{X_{I}} \rightarrow \cdots
$$

and

$$
\mathscr{O}_{X}^{*} \rightarrow \prod_{I,|I|=1} \mathscr{O}_{X_{I}}^{*} \rightarrow \prod_{I,|I|=2} \mathscr{O}_{X_{I}}^{*} \rightarrow \cdots
$$

Lemma 5.11. The complexes 5.10.1 and 5.10.2 are acyclic.

Proof. The complex 5.10.1 is the complex of coherent $\mathscr{O}_{X}$-modules associated to the complex of $\mathcal{R}$-modules $C$. Therefore 5.10 .1 is acyclic.

To see that 5.10 .2 is exact, note that if $\bar{x} \rightarrow X$ is a geometric point, then the stalk of 5.10 .2 at $\bar{x}$ is the complex $D_{\mathscr{O}_{X, \bar{x}}}$.

5.12. Turning now to the proof of 5.1, consider first the case when $G$ is a tame étale group scheme. In this case we prove the proposition by showing that any finite étale covering of $X^{\circ}$ is trivial.

For this, let $X_{i}(i=1, \ldots, s)$ denote the irreducible component defined by $x_{i}=0$, and for indices $i_{1}, \ldots, i_{t}$ define

$$
X_{i_{1} \cdots i_{t}}:=X_{i_{1}} \cap X_{i_{2}} \cap \cdots \cap X_{i_{t}} .
$$

By proper descent theory for étale morphisms, the category $\operatorname{Fet}(X)$ of finite étale $X$-schemes is equivalent to the category of collections $\left\{\left(P_{i} \rightarrow X_{i}, \sigma_{i j}\right)\right\}$, where $P_{i} \rightarrow X_{i}$ is a finite étale morphism and for every $i, j$

$$
\sigma_{i j}:\left.\left.P_{i}\right|_{X_{i j}} \rightarrow P_{j}\right|_{X_{i j}}
$$

is an isomorphism such that the diagrams over the triple intersections

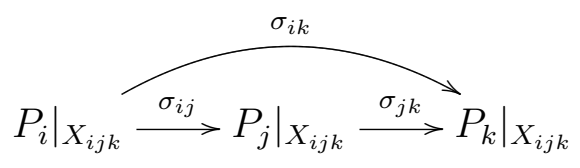

commute. 
Similarly if we set $X_{i}^{o}:=X_{i} \cap X^{o}$ etc., then giving a finite étale morphism $P^{o} \rightarrow X^{o}$ is equivalent to giving a collection of data $\left\{\left(P_{i}^{o} \rightarrow X_{i}^{o}\right), \sigma_{i j}^{o}\right\}$, where $P_{i}^{o} \rightarrow X_{i}^{o}$ is a finite étale morphism and

$$
\sigma_{i j}:\left.\left.P_{i}^{o}\right|_{X_{i j}^{o}} \rightarrow P_{j}^{o}\right|_{X_{i j}}
$$

is an isomorphism such that the analogue of 5.12 .3 commutes.

Now given such a collection of data $\left\{\left(P_{i}^{o} \rightarrow X_{i}^{o}\right), \sigma_{i j}^{o}\right\}$, let $P_{i} \rightarrow X_{i}$ be the normalization of $X_{i}$ in $P_{i}^{o}$. Since $X_{i}$ is regular and of dimension $\geq 2$ (by our assumption on $n$ ), the morphism $P_{i} \rightarrow X_{i}$ is étale. Furthermore, since $X_{i j}$ is normal the restriction functor

$$
\operatorname{Fet}\left(X_{i j}\right) \rightarrow \operatorname{Fet}\left(X_{i j}^{o}\right)
$$

is fully faithful. It follows that the $\sigma_{i j}^{o}$ extend uniquely to isomorphisms

$$
\sigma_{i j}:\left.\left.P_{i}\right|_{X_{i j}} \rightarrow P_{j}\right|_{X_{i j}}
$$

Furthermore, since the intersections $X_{i j k}$ are also normal the diagram 5.12 .3 commutes as its restriction to $X_{i j k}^{o}$ commutes.

We therefore obtain a collection of data $\left\{\left(P_{i} \rightarrow X_{i}\right), \sigma_{i j}\right\}$ inducing $\left\{\left(P_{i}^{o} \rightarrow X_{i}^{o}\right), \sigma_{i j}^{o}\right\}$.

5.13. For the case of general $G$, note first that by making a finite étale extension of $V$, we may assume that $G$ fits into an exact sequence

$$
1 \rightarrow \Delta \rightarrow G \rightarrow H \rightarrow 1
$$

where $\Delta$ is diagonalizable and $H$ is étale and tame.

Let us first show that the reduction functor 5.1.1 is fully faithful. Let $P_{1}$ and $P_{2}$ be two $G$-torsors over $X$, and set

$$
\bar{P}_{i}:=P_{i} / \Delta
$$

which is an $H$-torsor over $X$. Assume given an isomorphism of $G$-torsors

$$
\rho^{\circ}: P_{1}^{\circ} \rightarrow P_{2}^{\circ}
$$

over $X^{\circ}$. By the étale case, the induced isomorphism

$$
\bar{\rho}^{\circ}: \bar{P}_{1}^{\circ} \rightarrow \bar{P}_{2}^{\circ}
$$

extends uniquely to an isomorphism of $H$-torsors over $X$. Since the $\bar{P}_{i}$ are trivial $H$-torsors, the underlying scheme of $\bar{P}_{i}$ is isomorphic to a disjoint union of copies of $X$. Now to find a dotted arrow

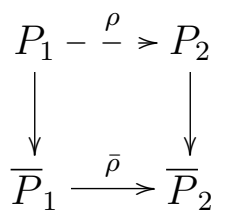

filling in the diagram and restricting to $\rho^{\circ}$, it suffices to find an extension of $\rho^{\circ}$ which is compatible with the $\Delta$-action (since the compatibility with the $G$-action can be verified after restriction to $X^{\circ}$ ). This reduces the proof of full faithfulness to the case when $G=\Delta$. Since $\Delta$ is equal to a finite product of copies of $\mu_{n}$ for various $n$, one further reduces to the case of $\Delta=\mu_{n}$ for some integer $n$. In this case the category of $\mu_{n}$-torsors (over either $X$ or $X^{\circ}$ ) is equivalent to the category of pairs $(L, \iota)$, where $L$ is a line bundle and $\iota$ is a trivialization of $L^{n}$. 
Let $P_{i}$ correspond to $\left(L_{i}, \iota_{i}\right)(i=1,2)$. The isomorphism $\rho^{\circ}$ corresponds to an isomorphism $\lambda: \mathscr{O}_{X^{\circ}} \rightarrow L_{2} \otimes L_{1}^{-1}$ such that the induced isomorphism

$$
\lambda^{\otimes n}: \mathscr{O}_{X^{\circ}} \rightarrow L_{2}^{\otimes n} \otimes L_{1}^{\otimes-n}
$$

is equal to $\iota_{2} \otimes \iota_{1}^{-1}$. The full faithfulness therefore follows from part (i) of the following lemma.

Lemma 5.14. (i) The restriction map

$$
\Gamma\left(X, \mathscr{O}_{X}^{*}\right) \rightarrow \Gamma\left(X^{\circ}, \mathscr{O}_{X^{\circ}}^{*}\right)
$$

is an isomorphism.

(ii) The restriction map

$$
H^{1}\left(X, \mu_{n}\right) \rightarrow H^{1}\left(X^{\circ}, \mu_{n}\right)
$$

is an isomorphism (here cohomology is taken with respect to the fppf-topology).

Proof. Statement (i) follows immediately from the exactness of 5.10 .2 (by 5.11), and the fact that the $X_{I}(I \neq \emptyset)$ are normal.

For (ii), define $X_{I}^{\circ}$ to be $X_{I} \cap X^{\circ}$. The spectral sequence of a filtered complex, applied to 5.10 .2 , gives a spectral sequence

$$
E_{1}^{p q}=\prod_{I,|I|=p} H^{q}\left(X_{I}, \mathscr{O}_{X_{I}}^{*}\right) \Longrightarrow H^{p+q}\left(X, \mathscr{O}_{X}^{*}\right)
$$

and similarly we have

$$
\widetilde{E}_{1}^{p q}=\prod_{I,|I|=p} H^{q}\left(X_{I}^{\circ}, \mathscr{O}_{X_{I}^{\circ}}^{*}\right) \Longrightarrow H^{p+q}\left(X^{\circ}, \mathscr{O}_{X^{\circ}}^{*}\right)
$$

Now observe that $H^{m}\left(E_{1}^{-0}\right)=0$ for $m \neq 0$, since $D_{\mathcal{R}}$ is acyclic, so this gives an isomorphism

$$
\operatorname{Pic}(X)=\operatorname{Ker}\left(\prod_{I,|I|=1} \operatorname{Pic}\left(X_{I}\right) \rightarrow \prod_{I,|I|=2} \operatorname{Pic}\left(X_{I}\right)\right),
$$

and also shows that there is an exact sequence

$$
0 \rightarrow H^{1}\left(\widetilde{E}_{1}^{-0}\right) \rightarrow \operatorname{Pic}\left(X^{\circ}\right) \rightarrow \operatorname{Ker}\left(\prod_{I,|I|=1} \operatorname{Pic}\left(X_{I}^{\circ}\right) \rightarrow \prod_{I,|I|=2} \operatorname{Pic}\left(X_{I}^{\circ}\right)\right)
$$

Now a direct calculation, using the fact that for $|I|=1$ the complement of $X_{I}^{\circ} \subset X_{I}$ has codimension $\geq 2$, shows that if $n>2$ then $H^{1}\left(\widetilde{E}_{1}^{-0}\right)$ is zero, and if $n=2$ we have $H^{1}\left(\widetilde{E}_{1}^{-0}\right) \simeq K^{*} / V^{*}$. In particular $H^{1}\left(\widetilde{E}_{1}^{-0}\right)$ is torsion free. Now for every $I$ with $|I|=1$ the map

$$
\operatorname{Pic}\left(X_{I}\right) \rightarrow \operatorname{Pic}\left(X_{I}^{\circ}\right)
$$

is an isomorphism since $X_{I}$ is regular. From the commutative diagram

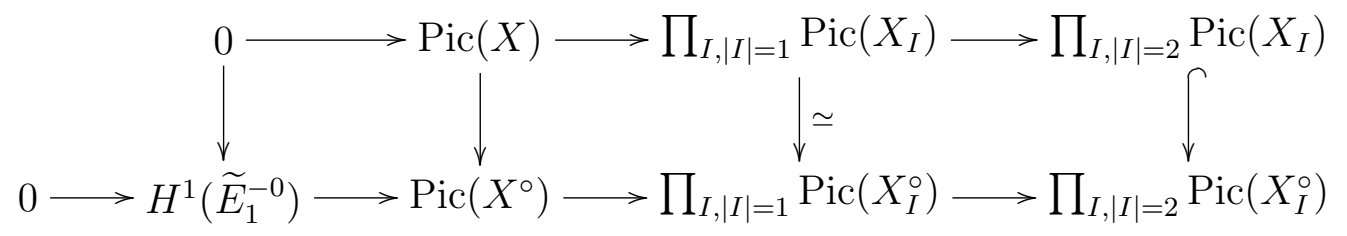


it then follows that the restriction map

$$
\operatorname{Pic}(X)_{\text {tors }} \rightarrow \operatorname{Pic}\left(X^{\circ}\right)_{\text {tors }}
$$

is an isomorphism.

To get statement (ii) in the lemma, consider now the short exact sequence

$$
0 \longrightarrow \mu_{n} \longrightarrow \mathbb{G}_{m} \stackrel{u \mapsto u^{n}}{\longrightarrow} \mathbb{G}_{m} \longrightarrow 0
$$

which induces a morphism of long exact sequences

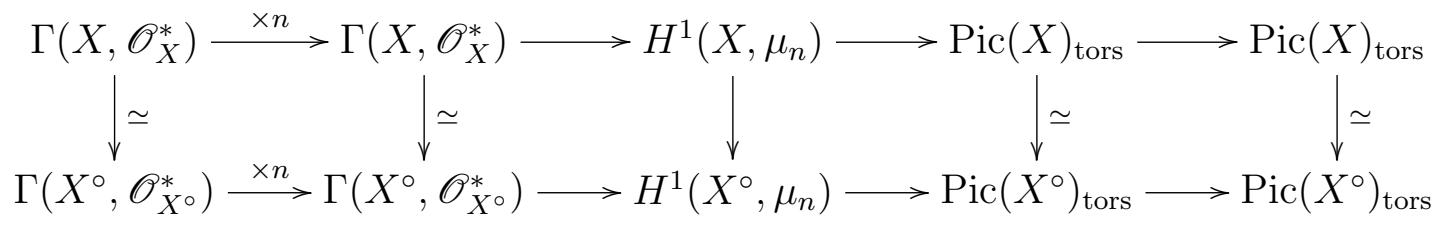

5.15. Returning finally to the proof of essential surjectivity in 5.1, note that by a similar reduction as in the start of the proof it suffices to consider the case when $G=\mu_{n}$. In this case the result follows from 5.14 (ii).

\section{COVERINGS OF TAME STACKS AND THEIR COARSE SPACES.}

6.1. Let $S$ be a scheme and let $\mathcal{X} / S$ be a tame stack. Let $\pi: \mathcal{X} \rightarrow X$ be the coarse moduli space. For every geometric point $\bar{x}: \operatorname{Spec}(k) \rightarrow \mathcal{X}$ let $G_{\bar{x}}$ denote the automorphism group scheme of $\bar{x}$ (a finite tame group scheme over $k$ ). If $L$ is a line bundle on $\mathcal{X}$, then the pullback $\bar{x}^{*} L$ is a rank 1 representation of $G_{\bar{x}}$.

Similarly, if $P \rightarrow \mathcal{X}$ is a finite étale morphism of stacks, then the fiber product $P_{\bar{x}}:=\bar{x} \times{ }_{\mathcal{X}} P$ is a finite disjoint union of copies of $\operatorname{Spec}(k)$ and there is a natural action of $G_{\bar{x}}$ on $P_{\bar{x}}$.

Proposition 6.2. The pullback functor

$$
\pi^{*}:(\text { line bundles on } X) \rightarrow(\text { line bundles on } \mathcal{X})
$$

induces an equivalence of categories between the category of line bundles on $X$ and the category of line bundles $L$ on $\mathcal{X}$ such that for every geometric point $\bar{x}: \operatorname{Spec}(k) \rightarrow \mathcal{X}$ the action of $G_{\bar{x}}$ on $\bar{x}^{*} L$ is trivial.

Proof. It is clear that if $M$ is a line bundle on $X$ and $L:=\pi^{*} M$, then for any geometric point $\bar{x}$ of $\mathcal{X}$ the action of $G_{\bar{x}}$ on $\bar{x}^{*} L$ is trivial. Therefore $\pi^{*}$ induces a functor between the indicated categories.

To see that $\pi^{*}$ is fully faithful, assume $M$ and $M^{\prime}$ are line bundles on $X$. Giving a map $M \rightarrow M^{\prime}$ is equivalent to giving a global section of $M^{-1} \otimes M^{\prime}$, and similarly giving a map $\pi^{*} M \rightarrow \pi^{*} M^{\prime}$ is equivalent to giving a global section of

$$
\pi^{*} M^{-1} \otimes_{\mathcal{O}_{\mathcal{X}}} \pi^{*} M^{\prime} \simeq \pi^{*}\left(M^{-1} \otimes_{\mathcal{O}_{X}} M^{\prime}\right)
$$

To prove the full faithfulness it therefore suffices to show that the natural map

$$
M^{-1} \otimes M^{\prime} \rightarrow \pi_{*} \pi^{*}\left(M^{-1} \otimes M^{\prime}\right)
$$


is an isomorphism. This can be verified locally on $X$, so it further suffices to consider the case when $M$ and $M^{\prime}$ are trivial. In this case it follows from the fact that $\mathcal{O}_{X} \rightarrow \pi_{*} \mathcal{O}_{\mathcal{X}}$ is an isomorphism (see for example $[\mathrm{AV}, 2.2 .1(5)]$ ).

For the essential surjectivity, let $L$ be a line bundle on $\mathcal{X}$ such that for every geometric point $\bar{x}: \operatorname{Spec}(k) \rightarrow \mathcal{X}$ the action of $G_{\bar{x}}$ on $\bar{x}^{*} L$ is trivial. We claim that in this case the sheaf $\pi_{*} L$ is a line bundle on $X$ and that the adjunction map

$$
\pi^{*} \pi_{*} L \rightarrow L
$$

is an isomorphism.

This can be verified locally in the flat topology on $X$. From this and [AVO, 3.2] we may assume that $X=\operatorname{Spec}(R)$ for some local ring $R$ and

$$
\mathcal{X}=[\operatorname{Spec}(A) / G]
$$

where $A$ is a finite local $R$-algebra and $G$ is a tame group scheme acting on $A$ such that the induced action on $A / \mathfrak{m}_{A} A$ is trivial. In this case $L$ corresponds to a free $A$-module $N$ of rank 1 with an action of $G$ compatible with the action on $A$. Moreover, the assumption that the group schemes $G_{\bar{x}}$ act trivially on the fibers $\bar{x}^{*} L$ implies that the action of $G$ on $N / \mathfrak{m}_{A} N$ is trivial. Since $G$ is linearly reductive, the map on $G$-invariants

$$
N^{G} \rightarrow\left(N / \mathfrak{m}_{A} N\right)^{G}
$$

is surjective. It follows that there exists an element $e \in N^{G}$ which is a basis for $N$ as an $A$-module. Then $\pi_{*} L$ corresponds to the $R$-module $A^{G} \cdot e=R \cdot e$ which is of rank 1 and the map

$$
A \otimes_{R} N^{G} \rightarrow N
$$

is an isomorphism.

Proposition 6.3. The pullback functor

$$
\pi^{*}: \operatorname{Fet}(X) \rightarrow \operatorname{Fet}(\mathcal{X})
$$

induces an equivalence of categories between $\operatorname{Fet}(X)$ and the category of finite étale morphisms of stacks $P \rightarrow \mathcal{X}$ such that for every geometric point $\bar{x}: \operatorname{Spec}(k) \rightarrow \mathcal{X}$ the action of $G_{\bar{x}}$ on $P_{\bar{x}}$ is trivial.

Proof. For the full faithfulness let $P_{1}$ and $P_{2}$ be two finite étale $X$-schemes. Let $\mathscr{M}$ or $\left(P_{1}, P_{2}\right)$ be the sheaf of sets on the étale site of $X$ given by

$$
(U \rightarrow X) \mapsto\left\{U \text {-morphisms } P_{1, U} \rightarrow P_{2, U}\right\} .
$$

Similarly define $\mathscr{M} \operatorname{or}\left(\pi^{*} P_{1}, \pi^{*} P_{2}\right)$ to be the sheaf on the étale site of $X$ (not $\mathcal{X}$ !) given by

$$
(U \rightarrow X) \mapsto\left\{\mathcal{X}_{U} \text {-morphisms }\left.\left.\pi^{*} P_{1}\right|_{\mathcal{X}_{U}} \rightarrow \pi^{*} P_{2}\right|_{\mathcal{X}_{U}}\right\}
$$

There is a natural map of sheaves

$$
\mathscr{M} \text { or }\left(P_{1}, P_{2}\right) \rightarrow \mathscr{M} \text { or }\left(\pi^{*} P_{1}, \pi^{*} P_{2}\right)
$$

which we need to show is an isomorphism. For this we may work locally on $X$, and hence may assume that both $P_{1}$ and $P_{2}$ are trivial in which case the result is immediate.

For the essential surjectivity let $P \rightarrow \mathcal{X}$ be a finite étale morphism such that for every geometric point $\bar{x}: \operatorname{Spec}(k) \rightarrow \mathcal{X}$ the action of $G_{\bar{x}}$ on $P_{\bar{x}}$ is trivial. 
By the full faithfulness already shown, to prove that $P$ is in the essential image of $\pi^{*}$ we may work fppf locally on $X$. By $[$ AVO, 3.2] we may therefore assume that

$$
\mathcal{X}=[\operatorname{Spec}(A) / G]
$$

as in the proof of 6.2 , and by a standard limit argument we may even assume that $R=A^{G}$ is strictly henselian local. In this case $A$ is also strictly henselian local and so the restriction of $P$ to $\operatorname{Spec}(A)$ is trivial. It follows that $\left.P\right|_{\operatorname{Spec}(A)}$ is isomorphic to

$$
\pi_{0}\left(\left.P\right|_{\operatorname{Spec}(A)}\right) \times \operatorname{Spec}(A)
$$

and so to show that $P$ is obtained by pullback from $\operatorname{Spec}(R)$ it suffices to show that the action of $G$ on $\pi_{0}\left(\left.P\right|_{\operatorname{Spec}(A)}\right)$ is trivial. This can be verified after base change to the closed point of $\operatorname{Spec}(A)$ in which case it holds by assumption.

6.4. Let $D / S$ be a tame finite group scheme. For a geometric point $\bar{x}: \operatorname{Spec}(k) \rightarrow \mathcal{X}$ we write $D_{\bar{x}}$ for the pullback of $D$ along the composite morphism

$$
\operatorname{Spec}(k) \stackrel{\bar{x}}{\longrightarrow} \mathcal{X} \longrightarrow S \text {. }
$$

If $P \rightarrow \mathcal{X}$ is a $D$-torsor over $\mathcal{X}$ then the pullback $\bar{x}^{*} P$ is a $D_{\bar{x}}$-torsor over $\operatorname{Spec}(k)$ which comes equipped with an action of $G_{\bar{x}}$, compatible with the action of $D_{\bar{x}}$.

Proposition 6.5. The pullback functor

$$
\pi^{*}:(D \text {-torsors on } X) \rightarrow(D \text {-torsors on } \mathcal{X})
$$

induces an equivalence of categories between the category of D-torsors on $X$ and the category of $D$-torsors $P \rightarrow \mathcal{X}$ on $\mathcal{X}$ such that for every geometric point $\bar{x}: \operatorname{Spec}(k) \rightarrow \mathcal{X}$ the action of $G_{\bar{x}}$ on $P_{\bar{x}}$ is trivial.

Proof. In the case when $D$ is an étale group scheme the result follows from 6.3.

If $D$ is diagonalizable the proposition can be seen as follows. Let $T$ be the Cartier dual of $D$ (a finite abelian group) and write

$$
T=\mathbb{Z} /\left(a_{1}\right) \times \cdots \times \mathbb{Z} /\left(a_{r}\right)
$$

for some positive integers $a_{i}$. Then the category of $D$-torsors on $X$ is equivalent to the category of collections $\left\{\left(L_{i}, \iota_{i}\right)\right\}_{i=1}^{r}$, where $L_{i}$ is a line bundle on $X$ and

$$
\iota_{i}: L_{i}^{a_{i}} \rightarrow \mathcal{O}_{X}
$$

is an isomorphism of line bundles. The category of $D$-torsors on $\mathcal{X}$ is described similarly. In the case when $D$ is diagonalizable 6.5 therefore follows from 6.2.

For the general case, we may work fppf locally on $S$ and therefore by [AVO, 2.20] may assume that $D$ fits into an exact sequence

$$
1 \rightarrow \Delta \rightarrow D \rightarrow H \rightarrow 1
$$

where $H$ is tame and étale and $\Delta$ is diagonalizable.

For the full faithfulness we proceed as in the proof of 6.3 . Let $P_{1}$ and $P_{2}$ be two $D$-torsors over $X$ and define $\mathscr{M}$ or $\left(P_{1}, P_{2}\right)$ (resp. $\mathscr{M}$ or $\left(\pi^{*} P_{1}, \pi^{*} P_{2}\right)$ ) to be the sheaf on the fppf site of $X$ which to any $U \rightarrow X$ associates the set of morphisms $P_{1, U} \rightarrow P_{2, U}$ of $D$-torsors over $U$ (resp. 
the set of morphisms $\left.\left.\pi^{*} P_{1}\right|_{\mathcal{X}_{U}} \rightarrow \pi^{*} P_{2}\right|_{\mathcal{X}_{U}}$ of $D$-torsors over $\left.\mathcal{X}_{U}\right)$. We then need to show that the map of fppf sheaves

$$
\mathscr{M} \text { or }\left(P_{1}, P_{2}\right) \rightarrow \mathscr{M} \text { or }\left(\pi^{*} P_{1}, \pi^{*} P_{2}\right)
$$

is an isomorphism. This is an fppf-local assertion so it suffices to consider the case when $P_{1}$ and $P_{2}$ are trivial. Fixing trivializations, the map on global sections defined by the map 6.5.1 is identified with the natural map

$$
\operatorname{Hom}_{S}(X, D) \rightarrow \operatorname{Hom}_{S}(\mathcal{X}, D)
$$

which is a bijection since $X$ is the coarse moduli space of $\mathcal{X}$.

For the essential surjectivity, let $P \rightarrow \mathcal{X}$ be a $D$-torsor such that for every geometric point $\bar{x}: \operatorname{Spec}(k) \rightarrow \mathcal{X}$ the action of $G_{\bar{x}}$ on $P_{\bar{x}}$ is trivial. We descend $P$ to a $D$-torsor on $X$ as follows. Let $\bar{P}$ denote the quotient of $P$ by the $\Delta$-action, so that $\bar{P}$ is an $H$-torsor over $\mathcal{X}$. Then by the case when $D$ is an étale group scheme there exists an $H$-torsor $\bar{Q} \rightarrow X$ such that $\bar{Q} \times_{X} \mathcal{X} \simeq \bar{P}$. Observe that $\bar{Q}$ is the coarse moduli space of $\bar{P}$ by [AV, $\left.2.2 .2(1)\right]$.

Now $P$ is a $\Delta$-torsor over $\bar{P}$ so by the case of a diagonalizable group scheme applied to the stack $\bar{P}$ we obtain a $\Delta$-torsor $Q \rightarrow \bar{Q}$ inducing $P$. Furthermore, by the full faithfulness already shown, the maps of $\Delta$-torsors

$$
\chi_{h}: Q_{h} \wedge P \rightarrow h^{*} P
$$

over $\bar{P}$ defined as in 3.3 , descend to maps of $\Delta$-torsors

$$
Q_{h} \wedge Q \rightarrow h^{*} Q
$$

giving $Q$ the structure of a $D$-torsor, inducing the $D$-torsor structure on $P$.

6.6. There is also a relative version of 6.5 .

Let $f: \mathscr{X} \rightarrow \mathscr{Y}$ be a morphism of tame stacks, and let

$$
\mathscr{X} \stackrel{\pi}{\longrightarrow} X_{\mathscr{Y}} \longrightarrow \mathscr{Y}
$$

be the relative moduli space (see for example [AVO2, §3]).

For any geometric point $\bar{x} \rightarrow \mathscr{X}$ we have a map of linearly reductive finite flat group schemes over $\bar{x}$

$$
\text { Aut }_{\mathscr{X}}(\bar{x}) \rightarrow \underline{\operatorname{Aut}}_{\mathscr{Y}}(f \circ \bar{x})
$$

Let $K_{\bar{x}}$ be the kernel of this homomorphism, so $K_{\bar{x}}$ is also a linearly reductive group scheme over $\bar{x}[\mathrm{AVO}, 2.7]$.

Now let $D$ be a linearly reductive group scheme over $S$ and let $P \rightarrow \mathscr{X}$ be a $D$-torsor. Then as in 6.4 there is an induced action of $K_{\bar{x}}$ on $P_{\bar{x}}$ compatible with the $D$-action.

Proposition 6.7. The functor

$$
\pi^{*}:\left(D \text {-torsors on } X_{\mathscr{Y}}\right) \rightarrow(D \text {-torsors on } \mathscr{X})
$$

induces an equivalence between the category of D-torsors on $X_{\mathscr{Y}}$ and the category of D-torsors $P$ on $\mathscr{X}$ such that for every geometric point $\bar{x} \rightarrow X$ the action of $K_{\bar{x}}$ on $P_{\bar{x}}$ is trivial. 
Proof. All the categories considered in the proposition are stacks over $\mathscr{Y}$ with the fppf topology. It therefore suffices to consider the case when $\mathscr{Y}$ is a scheme, in which case the result is 6.5 .

Proposition 6.8. With notation as in 6.6, let $P \rightarrow \mathscr{X}$ be a D-torsor. Then there exists an open substack $\mathscr{U} \subset \mathscr{X}$ such that a geometric point $\bar{x} \rightarrow \mathscr{X}$ factors through $\mathscr{U}$ if and only if the action of $K_{\bar{x}}$ on $P_{\bar{x}}$ is trivial.

Proof. Let $Y \rightarrow \mathscr{Y}$ be a smooth surjection with $\mathscr{Y}$ a scheme. Then it clearly suffices to prove the proposition for $\mathscr{X} \times_{\mathscr{Y}} Y \rightarrow Y$. We may therefore assume that $\mathscr{Y}=Y$ is a scheme. Let $\mathscr{X} \rightarrow X$ be the coarse moduli space. We may then further assume that $Y=X$.

Let $\mathscr{I} \rightarrow \mathscr{X}$ be the inertia stack of $\mathscr{X}$, and let $\mathscr{G}$ denote the automorphism group scheme over $\mathscr{X}$ of $P$. Since $P$ is a $D$-torsor the group scheme $\mathscr{G}$ over $\mathscr{X}$ is a twisted form of $D$. There is a canonical homomorphism of group schemes

$$
\rho: \mathscr{I} \rightarrow \mathscr{G} .
$$

We need to show that there exists an open substack $\mathscr{U} \subset \mathscr{X}$ such that for any geometric point $\bar{x} \rightarrow \mathscr{X}$ the homomorphism

$$
\rho_{\bar{x}}: \bar{x}^{*} \mathscr{I} \rightarrow \bar{x}^{*} \mathscr{G}
$$

is trivial if and only if $\bar{x}$ has image in $\mathscr{U}$.

This follows from the following lemma.

Lemma 6.9. Let $S$ be a noetherian scheme, let I/S be a finite (but not necessarily flat) group scheme such that for every point $s \in S$ the fiber $I_{s} \rightarrow \operatorname{Spec}(k(s))$ is linearly reductive, and let $G / S$ be a linearly reductive finite flat group scheme. Fix a homomorphism

$$
\rho: I \rightarrow G \text {. }
$$

Then the set of points $s \in S$ for which the homomorphism of group schemes over $k(s)$

$$
\rho_{s}: I_{s} \rightarrow G_{s}
$$

is trivial is an open subset of $S$.

Proof. We show that the set of points $s$ for which $\rho_{s}$ is trivial is constructible and stable under generization.

For the constructibility, note that since $I$ is finite over $S$ there exists a stratification of $S$ such that the restriction of $I$ to each stratum is finite and flat. It therefore suffices to consider the case when $I$ is finite and flat over $S$. In this case we claim that the set of points $s \in S$ for which $\rho_{s}$ is trivial is an open and closed subset of $S$.

For this note that we can without loss of generality replace $S$ by a faithfully flat covering, so we may assume that both $I$ and $G$ well-split in the sense of [AVO, 2.19]. Write

$$
I=\Delta \rtimes H, \quad G=\Sigma \rtimes \Gamma,
$$

where $\Delta$ and $\Sigma$ are diagonalizable of order a power of a prime $p$, and $H$ and $\Gamma$ are tame étale group schemes of order prime to $p$.

The homomorphism $\rho$ is then induced by homomorphisms

$$
\rho_{1}: \Delta \rightarrow \Sigma, \quad \rho_{2}: H \rightarrow \Gamma .
$$


The condition that $\rho_{2}$ is trivial is clearly an open and closed condition, and the condition that $\rho_{1}$ is trivial is equivalent to the condition that the map on Cartier duals

$$
\operatorname{Hom}\left(\Sigma, \mathbb{G}_{m}\right) \rightarrow \operatorname{Hom}\left(\Delta, \mathbb{G}_{m}\right)
$$

is trivial, which is also clearly an open and closed condition. This completes the proof of the constructibility of the set of points $s \in S$ for which $\rho_{s}$ is trivial.

For the stability under generization, we may without loss of generality assume that $S=$ $\operatorname{Spec}(V)$ is the spectrum of a discrete valuation ring. Let $\eta \in S$ (resp. $s \in S$ ) be the generic (resp. closed) point of $S$. We need to show that if $\rho_{s}$ is trivial, then $\rho_{\eta}$ is also trivial.

Let $I^{\prime} \subset I$ denote the scheme-theoretic closure of $I_{\eta}$ in $I$. Then $I^{\prime}$ is a finite flat group scheme over $S$ and $I^{\prime}$ is linearly reductive as the closed fiber of $I^{\prime}$ is a closed subgroup scheme of a linearly reductive group scheme. Moreover the map $I_{s}^{\prime} \rightarrow G_{s}$ is trivial since $\rho_{s}$ is trivial, and hence by the same reasoning used above it follows that $\rho_{\eta}$ is also trivial.

\section{TWISTED G-TORSORS AND STABILITY.}

In this section we introduce the notion of a stable twisted $G$-torsor, and define the stack ${ }_{G} \mathscr{K}_{X / S}$. The setup will be the following:

Setup 7.1. Let $\left(X, M_{X}\right) \rightarrow\left(S, M_{S}\right)$ be a $\log$ smooth special and essentially semistable morphism (in the sense of 2.2 ), with $X \rightarrow S$ proper.

7.2. Let $G / S$ be a tame finite flat group scheme. We define a twisted $G$-torsor on $X$ to be a pair $\left(j: M_{S} \hookrightarrow N_{S}, P\right)$, where $j$ is a simple extension and $P \rightarrow \mathcal{X}_{j}$ is a $G$-torsor on $\mathcal{X}_{j}$, where $\mathcal{X}_{j}$ is defined as in 2.2 .

7.3. The stack $\mathcal{X}_{j}$ has a well-defined singular locus defined as follows. On $\mathcal{X}_{j}$ there is a tautological simple morphism of $\log$ structures $\left.M_{X}\right|_{\mathcal{X}_{j}} \rightarrow N_{\mathcal{X}_{j}}$. The $\log$ structure $N_{\mathcal{X}_{j}}$ defines a canonical closed substack $\mathcal{X}_{j}^{s} \subset \mathcal{X}_{j}$, which we refer to as the singular substack. If $U \rightarrow \mathcal{X}_{j}$ is a morphism with $U$ a scheme and $\bar{x} \rightarrow U$ is a geometric point, then the image in $\mathcal{O}_{U, \bar{x}}$ of the ideal sheaf $I_{\mathcal{X}_{j}^{s}}$ of $\mathcal{X}_{j}^{s}$ can be described as follows. Choose for each irreducible element $e_{i} \in \bar{N}_{\mathcal{X}_{j}, \bar{x}}$ which is not in the image of $\bar{M}_{S, f(\bar{x})}$ a lifting $m_{i} \in\left(\left.N_{\mathcal{X}_{j}}\right|_{U}\right)_{\bar{x}}$ of $e_{i}$ and let $x_{i} \in \mathcal{O}_{U, \bar{x}}$ be the image of $m_{i}$. Assume that the $e_{i}$ 's are ordered as $e_{1}, \ldots, e_{s}$. Then the image of $I_{\mathcal{X}_{j}^{s}}$ in $\mathcal{O}_{U, \bar{x}}$ is the ideal generated by the elements

$$
x_{1} x_{2} \cdots \hat{x}_{j} \cdots x_{s}, \quad j=1, \ldots, s .
$$

Let $\mathcal{X}_{j}^{s o} \subset \mathcal{X}_{j}^{s}$ be the maximal open substack where the map $\mathcal{X}_{j}^{s} \rightarrow S$ is smooth.

7.4. A key notion in what follows will be the notion of a stable twisted $G$-torsor which we now define. This notion will be defined pointwise, so let us first discuss the definition over a separably closed field.

Assume that $S=\operatorname{Spec}(k)$ is the spectrum of a separably closed field and that $\left(j: M_{S} \hookrightarrow\right.$ $\left.N_{S}, P\right)$ is a twisted $G$-torsor. Let $Z \subset X$ be the singular locus of $X$ with the reduced structure. Étale locally $X$ is isomorphic to

$$
\operatorname{Spec}\left(k\left[x_{1}, \ldots, x_{n}\right] /\left(x_{1} \cdots x_{r}\right)\right)
$$

for some $r \leq n$, in which case $Z$ is the union of the closed subschemes defined by the ideals $\left(x_{i}, x_{j}\right)(1 \leq i, j \leq r)$. 
Let $Z_{1}, \ldots, Z_{t}$ be the connected components of $Z$. Recall (see the discussion in 2.2) that there is a canonical bijection between the connected components $Z_{i}$ of $Z$ and the irreducible elements in $\bar{M}_{S} \simeq \mathbb{N}^{r}$. This bijection is characterized by the condition that for any geometric point $\bar{z} \rightarrow Z_{i}$ the irreducible element $e_{i} \in \bar{M}_{S}$ is the unique irreducible element whose image in $\bar{M}_{X, \bar{z}}$ is not irreducible.

For each irreducible element $e_{i} \in \bar{M}_{S}$ there exists a unique integer $a_{i}$ such that the image of $e_{i}$ in $\bar{N}_{S}$ is equal to $a_{i}$ times an irreducible element of $\bar{N}_{S}$. In this way we associate to each connected component $Z_{i}$ an integer $a_{i}$.

On the other hand, consider a generic point $\bar{\eta} \rightarrow Z_{i}$ for some connected component $Z_{i}$ of $Z$. Then $\operatorname{Spec}\left(\mathcal{O}_{X, \bar{\eta}}\right)$ has exactly two irreducible components, say $W_{1}$ and $W_{2}$. Moreover, the rings $\mathcal{O}_{W_{p}, \bar{\eta}}$ are discrete valuation rings. Let $\pi_{p} \in \mathcal{O}_{W_{p}, \bar{\eta}}(p=1,2)$ be a uniformizer. Let $W_{p, \bar{\eta}}^{\circ} \subset W_{p, \bar{\eta}}$ be the generic point, and let

$$
P_{p}^{\circ} \rightarrow W_{p, \bar{\eta}}^{\circ}
$$

be the restriction of $P$. By 4.5 there exists a minimal integer $e_{\bar{\eta}, p}$ such that $P_{p}^{\circ}$ extends to

$$
\mathscr{W}_{p, e_{\bar{\eta}, p}}:=\left[\operatorname{Spec}\left(\mathcal{O}_{W_{p}, \bar{\eta}}[t] /\left(t^{e_{\bar{\eta}}, p}-\pi_{p}\right)\right) / \mu_{e_{\bar{\eta}, p}}\right] .
$$

Lemma 7.5. We have $e_{\bar{\eta}, 1}=e_{\bar{\eta}, 2}$.

Proof. Note that we have an isomorphism

$$
\mathscr{W}_{p, a_{i}} \simeq\left(W_{p} \times_{X} \mathscr{X}_{j}\right)_{\mathrm{red}}
$$

In particular the torsor $P \rightarrow \mathscr{X}_{j}$ restricts to a torsor $P_{p} \rightarrow \mathscr{W}_{p, a_{i}}$.

Therefore we must have $e_{\bar{\eta}, p} \mid a_{i}$. Let $f_{p}$ denote the ratio $a_{i} / e_{\bar{\eta}, p}$. We can then describe the integer $f_{p}$ as follows. The pullback of $\mathscr{W}_{p, a_{i}}$ to the closed point of $W_{p}$ is a nilpotent thickening of the classifying stack $B \mu_{a_{i}}$, so we have an inclusion

$$
B \mu_{a_{i}} \hookrightarrow \mathscr{W}_{p, a_{i}}
$$

Pulling back $P_{p}$ along this inclusion we obtain a $G$-torsor over $B \mu_{a_{i}}$ which corresponds to a conjugacy class of homomorphisms $\rho_{p}: \mu_{a_{i}} \rightarrow G$. The order of the kernel of this homomorphism depends only on the conjugacy class and is therefore a well-defined invariant. It follows from 6.7 that $f_{p}$ is equal to the order of this kernel.

Now observe that the two composite inclusions for $p=1,2$

$$
B \mu_{a_{i}} \hookrightarrow \mathscr{W}_{p, a_{i}} \hookrightarrow \mathscr{X}_{j} \times_{X} \operatorname{Spec}\left(\mathcal{O}_{X, \bar{\eta}}\right)
$$

differ by the automorphism of $B \mu_{a_{i}}$ given by $\zeta \mapsto \zeta^{-1}$. It follows that $f_{1}=f_{2}$, and therefore also $e_{\bar{\eta}, 1}=e_{\bar{\eta}, 2}$.

Remark 7.6. In what follows we write simple $e_{\bar{\eta}}$ for $e_{\bar{\eta}, 1}=e_{\bar{\eta}, 2}$.

Definition 7.7. We say that $\left(j: M_{S} \hookrightarrow N_{S}, P\right)$ is stable if for each connected component $Z_{i}$ of $Z$ the integer $a_{i}$ is equal to the least common multiple of the integers $e_{\bar{\eta}}$ as $\eta$ varies over the generic points of $Z_{i}$. 
Remark 7.8. Note that for each geometric point $\bar{\eta} \rightarrow Z$ mapping to a generic point of some connected component $Z_{i}$, the integer $e_{\bar{\eta}}$ is less than or equal to $|G|$. It follows that if $\left(j: M_{S} \hookrightarrow N_{S}, P\right)$ is stable then the integer $a_{i}$ is less than or equal to

$$
|G|^{\left\{\# \text { of irreducible components of } Z_{i}\right\}} \text {. }
$$

7.9. For a general base scheme $S$, a twisted $G$-torsor $\left(j: M_{S} \hookrightarrow N_{S}, P\right)$ over $S$ is stable if for every geometric point $\bar{s}$ the pullback of this twisted $G$-torsor to $\bar{s}$ is stable.

7.10. Let ${ }_{G} \mathscr{K}_{X / S}$ denote the stack over $S$ which to any $S$-scheme $T$ associates the groupoid of stable twisted $G$-torsors $\left(j:\left.M_{S}\right|_{T} \hookrightarrow N_{T}, P \rightarrow \mathcal{X}_{j}\right)$ over $T$. The main result, whose proof occupies the following sections, is then theorem 1.5.

\section{ReFormulation of the STABility CONDition.}

Let $\left(X, M_{X}\right) /\left(S, M_{S}\right)$ be as in 7.1 , and let $\left(j: M_{S} \hookrightarrow N_{S}, P\right)$ be a twisted $G$-torsor.

8.1. Assume first that $S=\operatorname{Spec}(k)$ is the spectrum of a separably closed field $k$. Let $\widetilde{X} \rightarrow X$ be the normalization of $X$, and let $\widetilde{Z} \subset \widetilde{X}$ be the preimage (with the reduced structure) of the singular locus $Z \subset X$. Note that $\widetilde{X}$ is smooth and that $\widetilde{Z}$ is a divisor with local normal crossings on $\widetilde{X}$.

Let $\left\{Z_{1}, \ldots, Z_{r}\right\}$ be the connected components of $Z$, and let $\widetilde{Z}_{i} \subset \widetilde{Z}$ be the preimage of $Z_{i}$ so that

$$
\widetilde{Z}=\widetilde{Z}_{1} \coprod \widetilde{Z}_{2} \cdots \coprod \widetilde{Z}_{r}
$$

Also let $\mathbb{N}^{r} \simeq \bar{M}_{S}$ be the isomorphism obtained by ordering the components $Z_{i}$, and let $\left\{a_{i}\right\}_{i=1}^{r}$ be the integers such that the map $\bar{M}_{S} \rightarrow \bar{N}_{S}$ is isomorphic to the map $\underline{a}: \mathbb{N}^{r} \rightarrow \mathbb{N}^{r}$.

Let $\widetilde{X}^{\circ} \subset \widetilde{X}$ denote the complement of $\widetilde{Z}$, and let $\widetilde{P}^{\circ} \rightarrow \widetilde{X}^{\circ}$ denote the pullback of $P$.

By 4.6 there exists a unique minimal set of integers $b_{1}, \ldots, b_{r}$ such that $\widetilde{P}^{\circ}$ extends to

$$
\widetilde{\mathscr{X}}_{\widetilde{Z}, \underline{b}}:=\widetilde{\mathscr{X}}_{\widetilde{Z}_{1}, b_{1}} \times{ }_{\widetilde{X}} \widetilde{\mathscr{X}}_{\widetilde{Z}_{2}, b_{2}} \times{ }_{\widetilde{X}} \cdots \times \times_{\widetilde{X}} \widetilde{\mathscr{X}}_{\widetilde{Z}_{r}, b_{r}} .
$$

Lemma 8.2. The twisted $G$-torsor $(j, P)$ is stable if and only if $a_{i}=b_{i}$ for all $i$.

Proof. For each geometric point $\bar{x} \rightarrow \widetilde{X}$ mapping to a generic point of $\widetilde{Z}_{i}$, the $\operatorname{ring} \mathscr{O}_{\widetilde{X}, \bar{x}}$ is a discrete valuation ring, and $P$ restricts to a $G$-torsor over the generic point of $\operatorname{Spec}\left(\mathscr{O}_{\widetilde{X}, \bar{x}}\right)$. By 4.2 we therefore have an integer $e_{\bar{x}}$ associated to $\bar{x}$. By 3.6 the integer $b_{i}$ is equal to the least common multiple of the integers $e_{\bar{x}}$ as $\bar{x}$ ranges over geometric generic points of $\widetilde{Z}_{i}$.

Now the integer $e_{\bar{x}}$ is also equal to the integer associated to the composite $\bar{x} \rightarrow \widetilde{X} \rightarrow X$ as in 7.4. This implies the lemma.

8.3. Next consider the case when $S=\operatorname{Spec}(V)$ is the spectrum of a discrete valuation ring and $M_{S}$ is induced by a chart $\mathbb{N}^{r} \rightarrow V$ sending all nonzero elements to 0 . Let $\eta$ (resp. $s$ ) denote the generic (resp. closed) point of $S$.

Lemma 8.4. The restriction to the closed fiber $\left(j_{s}, P_{s}\right)$ is stable if and only if the restriction to the generic fiber $\left(j_{\eta}, P_{\eta}\right)$ is stable. 
Proof. As above, let $\tilde{X}$ be the normalization of $X$, and let $Z_{1}, \ldots, Z_{r}$ be the connected components of the singular locus $Z \subset X$, ordered according to the isomorphism $\mathbb{N}^{r} \simeq \bar{M}_{S}$ coming from our chosen chart. Let $\widetilde{Z}_{i} \subset \widetilde{X}$ be the preimage of $Z_{i}$ with the reduced structure. For a sequence of integers $\underline{d}=\left(d_{1}, \ldots, d_{r}\right)$ set

$$
\widetilde{\mathscr{X}_{\widetilde{Z}, \underline{d}}}:=\widetilde{\mathscr{X}}_{\widetilde{Z}_{1}, d_{1}} \times{ }_{\widetilde{X}} \widetilde{\mathscr{X}_{\widetilde{Z}_{2}, d_{2}}} \times{ }_{\widetilde{X}} \cdots \times \times_{\widetilde{X}} \widetilde{\mathscr{X}}_{\widetilde{Z}_{r}, d_{r}} .
$$

Let $\underline{b}$ (resp. $\underline{b}^{\prime}$ ) be the minimal sequence of integers for which $\widetilde{P}_{\eta}^{\circ} \rightarrow \widetilde{X}_{\eta}^{\circ}$ (resp. $\widetilde{P}_{s}^{\circ} \rightarrow \widetilde{X}_{s}^{\circ}$ ) extends to $\widetilde{\mathscr{X}}_{\widetilde{Z}, \underline{b}, \eta}\left(\right.$ resp. $\left.\widetilde{\mathscr{X}}_{\widetilde{Z}, \underline{b}, s}\right)$. It then suffices to show that $\underline{b}=\underline{b}^{\prime}$.

By 5.1 the torsor $\widetilde{P}^{\circ}$ extends to $\widetilde{\mathscr{X}}_{\widetilde{Z}, \underline{b}}$, and therefore $b_{i}^{\prime} \mid b_{i}$ for all $i$. On the other hand, let $f_{i}$ denote $b_{i} / b_{i}^{\prime}$, and let

$$
\mathscr{Z}_{i} \subset \widetilde{\mathscr{X}_{\widetilde{Z}, \underline{b}}}
$$

be the smooth locus of the preimage of $\widetilde{Z}_{i}$ (with the reduced structure). The intertia stack $I_{\mathscr{Z}_{i}}$ is locally isomorphic to $\mu_{b_{i}}$. The $f_{i}$ define a well-defined closed subgroup $\mathscr{K}_{i} \subset I_{\mathscr{Z}_{i}}$ of the inertia stack of $\mathscr{Z}_{i}$, and the restriction of $P$ defines a conjugacy class of homomorphisms $\mathscr{K}_{i} \rightarrow G$. The restriction to the closed fiber of this conjugacy class of homomorphisms is the zero class, and since $\mathscr{K}_{i}$ and $G$ are tame group schemes it follows that it is zero over all of $\mathscr{Z}_{i}$ (see 6.9). From this we conclude that $f_{i}=1$ for all $i$.

8.5. A similar argument also shows the following. Again assume that $S=\operatorname{Spec}(V)$ is the spectrum of a discrete valuation ring, but we do not assume that there exists a chart $\mathbb{N}^{r} \rightarrow V$ sending all nonzero elements to zero (so in particular the closed fiber could have more singular components than the generic fiber).

Lemma 8.6. If the restriction to the closed fiber $\left(j_{s}, P_{s}\right)$ is stable then the generic fiber $\left(j_{\eta}, P_{\eta}\right)$ is also stable.

Proof. After possibly replacing $V$ by a finite extension, we may assume that there exists a chart $\mathbb{N}^{r} \rightarrow V$ for $M_{S}$ sending all nonzero elements to the maximal ideal. Let $Z_{i} \subset X$ be the connected component of the singular locus corresponding to the $i$-th standard generator of $\mathbb{N}^{r}$.

After possibly reordering the generators of $\mathbb{N}^{r}$ we may assume that $Z_{1}, \ldots, Z_{s}$ have nonempty generic fibers (for some $s \leq r$ ), and that $Z_{s+1}, \ldots, Z_{r}$ are contained in the closed fiber.

For $1 \leq i \leq s$, let $b_{i}$ (resp. $b_{i}^{\prime}$ ) denote the least common multiple of the integers $e_{\bar{x}}$ as $\bar{x}$ varies over geometric generic points of the generic (resp. closed) fiber of $Z_{i}$. Then the proof of 8.4 shows that $b_{i}=b_{i}^{\prime}$. If the closed fiber $\left(j_{s}, P_{s}\right)$ is stable then $a_{i}=b_{i}^{\prime}$ for all $i \leq s$ and therefore $b_{i}=a_{i}$ also. This implies that the generic fiber of $(j, P)$ is stable.

Corollary 8.7. For arbitrary $S$ there exists an open subset $U \subset S$ such that a geometric point $\bar{s} \rightarrow S$ has image in $U$ if and only if the fiber of $(j, P)$ over $\bar{s}$ is stable.

Proof. Combining 8.4 and 8.6 one sees that the set of points $s \in S$ for which the fiber of $(j, P)$ is stable is a constructible set stable under generization, and hence open. 


\section{Algebraicity of ${ }_{G} \mathscr{K}_{X / S}$}

Let $\left(X, M_{X}\right) /\left(S, M_{S}\right)$ be as in 7.1 .

9.1. Let $\mathscr{S} \rightarrow S$ be the stack classifying simple extensions $j: M_{S} \hookrightarrow N$ as in [AVO2, A.6]. As in loc. cit. the stack $\mathscr{S}$ is an algebraic stack. The universal simple extension over $\mathscr{S}$ then defines an algebraic stack $\mathcal{X}_{\mathscr{S}} \rightarrow X_{\mathscr{S}}$ over $\mathscr{S}$. Now consider the relative hom-stack

$$
\mathscr{H}:=\underline{\operatorname{Hom}}_{\mathscr{S}}\left(\mathcal{X}_{\mathscr{S}}, B G\right)
$$

over $\mathscr{S}$. By $[\mathrm{AVO} 2, \mathrm{C} .4]$ the stack $\mathscr{H}$ is algebraic locally of finite type over $\mathscr{S}$ and the diagonal

$$
\mathscr{H} \rightarrow \mathscr{H} \times \mathscr{S} \mathscr{H}
$$

is quasi-compact and separated.

The stack ${ }_{G} \mathscr{K}_{X / S}$ is a substack of $\mathscr{H}$, which is an open substack by 8.7. In particular ${ }_{G} \mathscr{K}_{X / S}$ is algebraic.

9.2. By the same argument as in the proof of $[\mathrm{Ol} 2,1.11]$, for any integer $N$ there is a quasicompact open substack $\mathscr{S}^{\leq N} \subset \mathscr{S}$ classifying simple extensions $M_{S} \hookrightarrow N_{S}$ such that for every geometric point $\bar{s} \rightarrow S$ the order of

$$
\operatorname{Coker}\left(\bar{M}_{S, \bar{s}}^{\mathrm{gp}} \rightarrow \bar{N}_{S, \bar{s}}^{\mathrm{gp}}\right)
$$

is less than or equal to $N$. Let $M$ be an integer such that for any geometric point $\bar{s} \rightarrow S$ the number of irreducible components of the singular locus of $X_{\bar{s}}$ is less than or equal to $M$. Then it follows from 7.8 that ${ }_{G} \mathscr{K}_{X / S}$ has image in $\mathscr{S}^{\leq N}$, where $N=(\# G)^{M}$.

In particular the stack ${ }_{G} \mathscr{K}_{X / S}$ is quasi-compact and hence of finite type over $S$.

Lemma 9.3. The diagonal of ${ }_{G} \mathscr{K}_{X / S}$ is quasi-finite.

Proof. It suffices to show that if $k$ is an algebraically closed field, $M_{k}$ is a log structure on $k$, and $\left(j: M_{k} \hookrightarrow N_{k}, P \rightarrow \mathscr{X}_{j}\right)$ is an object of ${ }_{G} \mathscr{K}_{X / S}$, then the automorphism group of this object is finite. Since the automorphism group of $N_{k}$ over $M_{k}$ is finite, it further suffices to show that the automorphism group of the $G$-torsor $P$ on $\mathscr{X}_{j}$ is finite. Write $G$ as an extension

$$
1 \rightarrow \Delta \rightarrow G \rightarrow H \rightarrow 1
$$

where $\Delta$ is diagonalizable and $H$ is tame and étale. Let $\bar{P}$ denote the $H$-torsor $P / \Delta$. The sheaf on $\mathscr{X}_{j}$ of automorphisms of $\bar{P}$, which commute with the $H$-action, is a locally constant sheaf $\mathscr{H}$ locally isomorphic to $H$. Furthermore, since $\Delta$ is abelian the automorphism group of $P$ over $\bar{P}$ is isomorphic to $\Delta$. To prove the lemma, it therefore suffices to prove the following two statements:

(a) Let $\mathscr{X} / k$ be a proper algebraic stack, and let $\mathscr{H}$ be a locally constant sheaf of finite groups on $\mathscr{X}$. Then $\Gamma(\mathscr{X}, \mathscr{H})$ is a finite set.

(b) Let $\mathscr{Y} / k$ be a proper reduced algebraic stack. Then the set of maps $\mathscr{Y} \rightarrow \Delta$ is finite.

For (a), let $p: Y \rightarrow \mathscr{X}$ be a proper surjection with $Y$ a scheme (such a morphism exists by Chow's lemma $[\mathrm{Ol} 3,1.1])$. Then the map

$$
\Gamma(\mathscr{X}, \mathscr{H}) \rightarrow \Gamma\left(Y, p^{*} \mathscr{H}\right)
$$


is injective. It therefore suffices to prove (a) in the case when $\mathscr{X}$ is a scheme, in which case it follows from [SGA4, XIV, 1.1].

For $(b)$ note that any morphism $\mathscr{Y} \rightarrow \Delta$ factors uniquely through $\operatorname{Spec}\left(\Gamma\left(\mathscr{Y}, \mathscr{O}_{\mathscr{Y}}\right)\right)$. Since $\mathscr{Y}$ is assumed reduced, the $\operatorname{scheme} \operatorname{Spec}\left(\Gamma\left(\mathscr{Y}, \mathscr{O}_{\mathscr{Y}}\right)\right)$ is a finite disjoint union of copies of $\operatorname{Spec}(k)$. Statement (b) therefore follows from the fact that $\Delta(k)$ is finite.

Proposition 9.4. The stack $\mathscr{K}_{X / S}$ is an Artin stack of finite type over $S$ with finite diagonal.

Proof. It remains to verify the valuative criterion for properness for the diagonal of ${ }_{G} \mathscr{K}_{X / S}$.

So assume that $S=\operatorname{Spec}(V)$ is the spectrum of a discrete valuation $\operatorname{ring} V$ and let $\pi \in V$ be a uniformizer. We may also assume that we have a chart $\mathbb{N}^{r} \rightarrow M_{S}$ defining an isomorphism $\mathbb{N}^{r} \simeq \bar{M}_{S, \bar{s}}$ (where $s \in S$ is the closed point). Observe then that for any simple extension $M_{S} \hookrightarrow N_{S}$ we obtain an isomorphism $\bar{N}_{S, \bar{s}} \simeq \mathbb{N}^{r}$ and a sequence of integers $a_{1}, \ldots, a_{r}$ such that the map $\bar{M}_{S, \bar{s}} \rightarrow \bar{N}_{S, \bar{s}}$ is identified with the map

$$
\cdot \underline{a}: \mathbb{N}^{r} \rightarrow \mathbb{N}^{r}
$$

Now assume given two simple extensions $j: M_{S} \hookrightarrow N_{S}$ and $j^{\prime}: M_{S} \hookrightarrow N_{S}^{\prime}$ which become isomorphic over the generic fiber, and let $P \rightarrow \mathscr{X}_{j}$ and $P^{\prime} \rightarrow \mathscr{X}_{j^{\prime}}$ be two stable $G$-torsors whose restriction to $\mathscr{X}_{j, \eta} \simeq \mathscr{X}_{j^{\prime}, \eta}$ are equal. We claim that then there is an isomorphism of $\log$ structures $\tau: N_{S} \rightarrow N_{S}^{\prime}$ such that the triangle

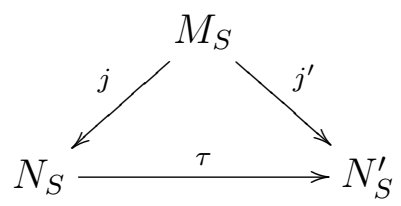

commutes. Let $\underline{a}$ (resp. $\underline{a}^{\prime}$ ) be the sequence of integers obtained from $j$ (resp. $j^{\prime}$ ) as above. To prove that $j$ is isomorphic to $j^{\prime}$ it suffices to show that $a_{i}=a_{i}^{\prime}$ for all $i$.

This can be verified on the closed fiber $X_{s}$ of $X$, where it follows from the definition of stable (note that the restrictions $P_{\bar{s}}^{\circ} \rightarrow X_{\bar{s}}^{\circ}$ and $P_{\bar{s}}^{\prime \circ} \rightarrow X_{\bar{s}}^{\circ}$ are isomorphic, as after making a ramified base change $V \rightarrow V^{\prime}$ the two torsors $P^{\circ} \rightarrow X^{\circ}$ and $P^{\prime \circ} \rightarrow X^{\circ}$ become isomorphic by 4.3$)$.

Fix now a simple extension $j: M_{S} \hookrightarrow N_{S}$ with corresponding sequence of integers $\underline{a}$.

Let $f_{1}, \ldots, f_{r} \in V$ be the images of the standard generators of $\mathbb{N}^{r}$ under the composite $\mathbb{N}^{r} \rightarrow M_{S} \rightarrow V$. Then the stack classifying simple extensions $M_{S} \hookrightarrow N$, such that the induced $\operatorname{map} \bar{M}_{S, \bar{s}} \rightarrow \bar{N}_{\bar{s}}$ is given by 9.4 .1 , is isomorphic to

$$
\left[\operatorname{Spec}\left(V\left[x_{1}, \ldots, x_{r}\right] /\left(x_{i}^{a_{i}}-f_{i}\right)_{i=1}^{r} / \mu_{a_{1}} \times \cdots \times \mu_{a_{r}}\right] .\right.
$$

In particular this is a proper stack over $V$.

It follows that to verify the properness of the diagonal of ${ }_{G} \mathscr{K}_{X / S}$ it suffices to consider the following situation.

Assume given a simple extension $j: M_{S} \hookrightarrow N_{S}$ and let $\mathcal{X}_{j} \rightarrow X$ be the resulting stack. Let $P_{i} \rightarrow \mathcal{X}_{j}(i=1,2)$ be two $G$-torsors, and assume given an isomorphism $\iota_{\eta}: P_{1, \eta} \rightarrow P_{2, \eta}$ over the generic point $\eta \in S$. We then must show that $\iota_{\eta}$ extends uniquely to an isomorphism $\iota: P_{1} \rightarrow P_{2}$. 
By the uniqueness we may work étale locally on $S$, so we may assume that $G$ is equal to an extension

$$
1 \rightarrow \Delta \rightarrow G \rightarrow H \rightarrow 1
$$

where $\Delta$ is diagonalizable and $H$ is étale. Let $P_{i, H}$ denote $P_{i} / \Delta$, so $P_{i, H}$ is an $H$-torsor and the projection $P_{i} \rightarrow P_{i, H}$ realizes $P_{i}$ as a $\Delta$-torsor over $P_{i, H}$.

By a standard limit argument it suffices to construct the extension of $\iota_{\eta}$ over the base change of $\mathcal{X}_{j}$ to the strict henselization of $X$ at a geometric point in the closed fiber. As in 2.5 we can write such a base change as

$$
[\operatorname{Spec}(R) / D]
$$

where $R$ is either equal to the strict henselization at the point $z_{1}=\cdots=z_{r}=0$ of

$$
V\left[z_{1}, \ldots, z_{r}\right] /\left(z_{1} \cdots z_{s}=\pi^{a}\right)
$$

for some $a \neq 0$, or the strict henselization of

$$
V\left[z_{1}, \ldots, z_{r}\right] /\left(z_{1} \cdots z_{s}=0\right) .
$$

Now any extension of $\iota_{\eta}$ over $\operatorname{Spec}(R)$ is automatically compatible with the $D$-action, so to construct the extension of $\iota_{\eta}$ it suffices to construct an extension over $\operatorname{Spec}(R)$.

First observe that the isomorphism $P_{1, H, \eta} \rightarrow P_{2, H, \eta}$ defined by $\iota_{\eta}$ extends uniquely to an isomorphism $P_{1, H} \rightarrow P_{2, H}$ over $\operatorname{Spec}(R)$. In the case when $R$ is of the form 9.4.2 this is immediate as $R$ is normal, which implies that $P_{i, H}$ is equal to the normalization of $\operatorname{Spec}(R)$ in $P_{i, H, \eta}(i=1,2)$. In the case when $R$ is of the form 9.4 .3 note that the category of torsors on $\operatorname{Spec}(R)$ is equivalent to the category of collections $\left(P_{i}, \sigma_{i j}\right)$, where $P_{i}$ is a torsor over $\operatorname{Spec}\left(R /\left(z_{i}\right)\right)(i=1, \ldots, s)$, and $\sigma_{i j}:\left.\left.P_{i}\right|_{R /\left(z_{i}, z_{j}\right)} \rightarrow P_{j}\right|_{R /\left(z_{i}, z_{j}\right)}$ is an isomorphism of torsors over $\operatorname{Spec}\left(R /\left(z_{i}, z_{j}\right)\right)$ satisfying a suitable cocycle condition over the triple overlaps (as in the proof of 5.1). Since the rings $R /\left(z_{i}\right)$ and $R /\left(z_{i}, z_{j}\right)$ are normal, the isomorphisms over the generic fiber extend uniquely.

Now observe that $P_{i, H}$ is isomorphic to a finite disjoint union of copies of $\operatorname{Spec}(R)$, so to lift this isomorphism $P_{1, H} \rightarrow P_{2, H}$ to the $P_{i}$ we are reduced to the case when $G$ is diagonalizable. In this case $G$ is isomorphic to a finite product of schemes of the form $\mu_{n}$ (for various $n$ ), which further reduces the proof to the case $\mu_{n}$. In this case $P_{i}$ corresponds to a pair $\left(L_{i}, \gamma_{i}\right)$, where $L_{i}$ is a line bundle and $\gamma_{i}: L_{i}^{n} \rightarrow R$ is an isomorphism. Let $M$ denote $L_{1} \otimes L_{2}^{-1}$. Then $M$ comes equipped with a trivialization $f \in M^{\otimes n}$ as well as a trivialization $g_{\eta} \in M_{\eta}$ such that $g_{\eta}^{n}$ is equal to the restriction of $f$. The claim is then that $g_{\eta}$ extends to a trivialization of $M$.

In the case when $R$ is of the form 9.4.2 this is immediate as $R$ is normal. In the case when $R$ has the form 9.4 .3 note that $R$ is the equalizer of the two maps

$$
\prod_{i=1}^{s} R /\left(z_{i}\right) \rightrightarrows \prod_{1 \leq i<j \leq s} R /\left(z_{i}, z_{j}\right) \text {. }
$$

Tensoring this sequence with $M$ we therefore obtain an equalizer diagram

$$
0 \rightarrow M \rightarrow \prod_{i=1}^{s} M / z_{i} M \rightrightarrows \prod_{1 \leq i<j \leq s} M /\left(z_{i}, z_{j}\right) M
$$


Let $g_{\eta}^{i} \in\left(M / z_{i} M\right)_{\eta}$ denote the image of $g_{\eta}$. Since $R /\left(z_{i}\right)$ is normal we in fact have $g_{\eta}^{i} \in$ $M / z_{i} M$. Moreover, since $R /\left(z_{i}, z_{j}\right)$ is normal the collection $\left(g_{\eta}^{i}\right)$ lies in the equalizer of the two maps

$$
\prod_{i=1}^{s} M / z_{i} M \rightrightarrows \prod_{1 \leq i<j \leq s} M /\left(z_{i}, z_{j}\right) M
$$

as this can be verified on the generic fiber. Therefore $g_{\eta}$ is in $M$. This completes the proof that the diagonal of ${ }_{G} \mathscr{K}_{X / S}$ is finite.

\section{StabilizATiON}

10.1. Let $\left(X, M_{X}\right) /\left(S, M_{S}\right)$ be as in 7.1, let $j: M_{S} \hookrightarrow N_{S}$ be a simple extension, and let $P \rightarrow \mathscr{X}_{j}$ be a twisted $G$-torsor. We explain in this section how to construct a subextension

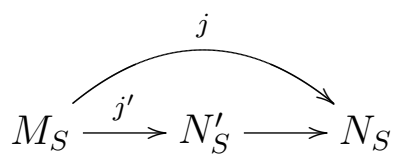

such that $P$ descends to a $G$-torsor $P^{\prime} \rightarrow \mathscr{X}_{j^{\prime}}$ which is stable.

10.2. Let us begin by considering the case when $S=\operatorname{Spec}(k)$ is the spectrum of a separably closed field $k$.

Fix an ordering $Z_{1}, \ldots, Z_{r}$ of the connected components of the singular locus of $X$, and recall that this defines isomorphisms

$$
\mathbb{N}^{r} \rightarrow \bar{M}_{S}, \quad \mathbb{N}^{r} \rightarrow \bar{N}_{S}
$$

such that we have a commutative diagram

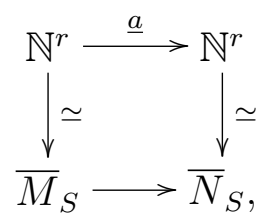

for some sequence of positive integers $\underline{a}=\left(a_{1}, \ldots, a_{r}\right)$.

On the other hand, for any geometric generic point $\bar{\eta} \rightarrow Z_{i}$ we obtain by 4.2 an integer $e_{\bar{\eta}}$, and we let $b_{i}$ denote the least common multiple of the integers $e_{\bar{\eta}}$ as $\bar{\eta}$ ranges over generic points of $Z_{i}$. By the definition of the integers $e_{\bar{\eta}}$ we have $b_{i} \mid a_{i}$. Let $f_{i}$ denote $a_{i} / b_{i}$.

Define $N_{S}^{\prime} \subset N_{S}$ to be the fiber product of the diagram

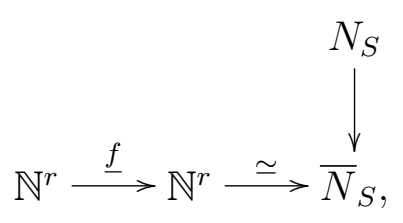

so we have a diagram of simple extensions

$$
M_{S} \stackrel{j^{\prime}}{\longrightarrow} N_{S}^{\prime} \longrightarrow N_{S}
$$


10.3. There is a natural morphism of stacks

$$
\pi^{\prime}: \mathscr{X}_{j} \rightarrow \mathscr{X}_{j^{\prime}}
$$

defined as follows.

Let $g: Y \rightarrow X$ be a morphism of schemes, and suppose given an object

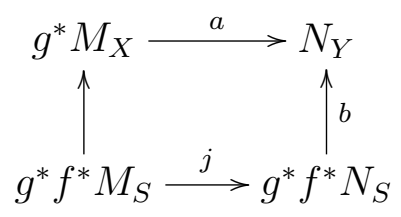

of $\mathscr{X}_{j}(Y)$. We claim that there exists a unique sub-log structure $N_{Y}^{\prime} \subset N_{Y}$ containing the images of $g^{*} M_{X}$ and $g^{*} f^{*} N_{S}^{\prime}$ such that the resulting diagram

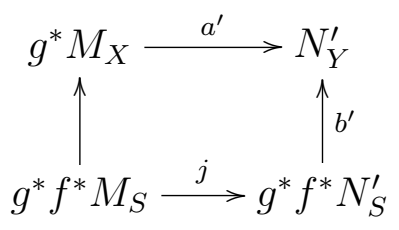

is an object of $\mathscr{X}_{j^{\prime}}(Y)$. The functor $\pi^{\prime}$ is defined by sending 10.3.1 to 10.3.2.

For this note first that $N_{Y}^{\prime}$ is determined by the subsheaf of monoids $\bar{N}_{Y}^{\prime} \subset \bar{N}_{Y}$, and this subsheaf is in turn determined by its stalks. Now for a geometric point $\bar{y}$ we can identify the diagram of stalks

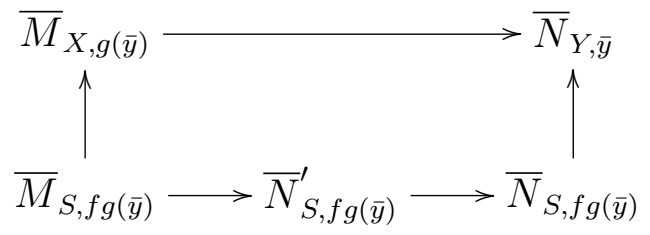

with the diagram

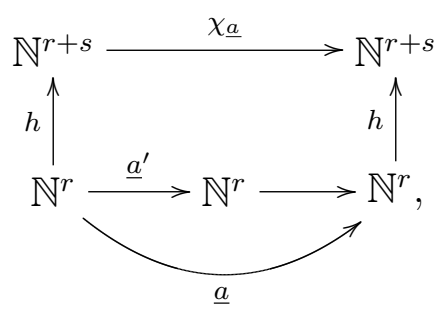

where $h$ and $\chi_{\underline{a}}$ are defined as in 2.4, and $\underline{a}$ and $\underline{a}^{\prime}$ are collections of integers with $a_{i}^{\prime} \mid a_{i}$ for all $i$. Let $F_{\bar{y}} \subset \bar{N}_{Y, \bar{y}} \simeq \mathbb{N}^{r+s}$ be the submonoid generated by the elements $a_{i}^{\prime} e_{i}(i<r)$ and $a_{r}^{\prime} e_{i}$ $(i \geq r)$. Then we want a sub-log structure $N_{Y}^{\prime} \subset N_{Y}$ such that $\bar{N}_{Y^{\prime}, \bar{y}}=F_{\bar{y}}$ for all $\bar{y} \rightarrow Y$.

From this the uniqueness follows, and also it suffices to construct $N_{Y}^{\prime}$ étale locally on $X$. Furthermore, it suffices to construct $N_{Y}^{\prime}$ in the universal case, over $\mathscr{X}_{j}$.

We can therefore assume that $\mathscr{X}_{j}$ is given by a fiber product as in 2.5. Let

$$
\tau: \mathbb{N}^{r} \rightarrow \mathbb{N}^{r}
$$

be the map sending $e_{i}$ to $\left(a / a_{i}^{\prime}\right) e_{i}$, and let

$$
\xi: \mathbb{N}^{r+s} \rightarrow \mathbb{N}^{r+s}
$$


be the map sending $e_{i}$ to $\left(a_{i} / a_{i}^{\prime}\right) e_{i}$ for $i<r$ and $\left(a_{r} / a_{r}^{\prime}\right) e_{i}$ for $i \geq r$. Also let $\Delta^{\prime}$ denote the kernel of the map

$$
\prod_{i=0}^{s} \mu_{a_{r}^{\prime}} \rightarrow \mu_{a_{r}^{\prime}}, \quad\left(\zeta_{0}, \ldots, \zeta_{s}\right) \mapsto \zeta_{0} \cdots \zeta_{s} .
$$

We then have a commutative diagram

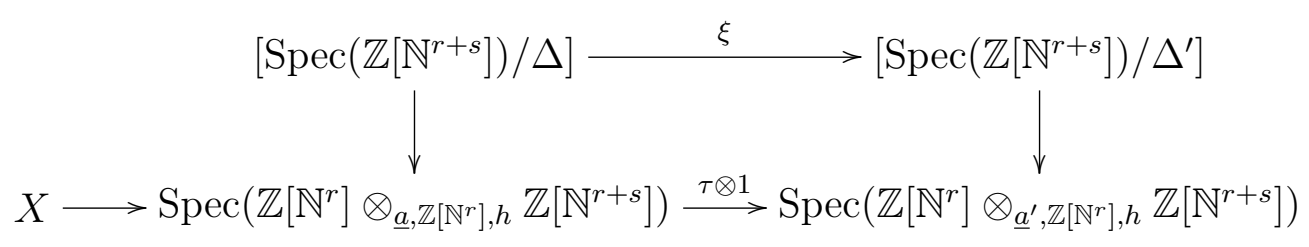

which defines a morphism

$$
\pi^{\prime}: \mathscr{X}_{j} \rightarrow \mathscr{X}_{j^{\prime}}
$$

by base changing to $X$. By construction there is a natural map $\pi^{*} N_{\mathscr{X}_{j^{\prime}}} \rightarrow N_{\mathscr{X}_{j}}$ of tautological log structures, which has the desired properties on stalks.

Remark 10.4. The local description of the map $\pi^{\prime}: \mathscr{X}_{j} \rightarrow \mathscr{X}_{j^{\prime}}$ in terms of the diagram 10.3.3 also shows that the relative coarse moduli space of $\mathscr{X}_{j} \rightarrow \mathscr{X}_{j^{\prime}}$ is equal to $\mathscr{X}_{j^{\prime}}$.

10.5. We claim that $P$ descends to a torsor $P^{\prime} \rightarrow \mathscr{X}_{j^{\prime}}$. By construction this torsor $P^{\prime}$ will be stable.

For a geometric point $\bar{x} \rightarrow \mathscr{X}_{j}$ let $K_{\bar{x}}$ denote the kernel of the map of group schemes

$$
\text { Aut }_{\mathscr{X}_{j}}(\bar{x}) \rightarrow \text { Aut }_{\mathscr{X}_{j^{\prime}}}(\pi \circ \bar{x}) .
$$

By 6.7 it suffices to show that for every geometric point $\bar{x} \rightarrow \mathscr{X}_{j}$ the action of $K_{\bar{x}}$ on $P_{\bar{x}}$ is trivial.

10.6. Assume that $\bar{x}$ has image in $Z_{i}$, and let $\bar{y} \rightarrow X$ be the composite $\bar{x} \rightarrow \mathscr{X}_{j} \rightarrow X$. Write the strict henselization $\mathcal{O}_{X, \bar{x}}$ as

$$
\mathcal{O}_{X, \bar{x}} \simeq\left(k\left[x_{1}, \ldots, x_{n}\right] /\left(x_{1} \cdots x_{s}\right)\right)^{\mathrm{sh}},
$$

where the right side denotes the strict henselization at the origin. For an integer $c$ let $H_{c} \subset \mu_{c}^{s}$ denote the kernel of the sum map

$$
\mu_{c}^{s} \rightarrow \mu_{c}, \quad\left(\zeta_{1}, \ldots, \zeta_{s}\right) \mapsto \prod_{i=1}^{s} \zeta_{i}
$$

and let $\mathscr{V}_{c}$ denote the stack quotient

$$
\mathscr{V}_{c}:=\left[\operatorname{Spec}\left(k\left[z_{1}, \ldots, z_{n} /\left(z_{1} \cdots z_{s}\right)\right) / H_{c}\right],\right.
$$

where $\underline{\zeta} \in H_{c}$ acts by

$$
z_{i} \mapsto \zeta_{i} z_{i} \quad(i \leq s), \quad z_{i} \mapsto z_{i} \quad(i>s)
$$

Then by construction we have

$$
\mathscr{X}_{j} \times_{X} \operatorname{Spec}\left(\mathcal{O}_{X, \bar{x}}\right) \simeq \mathscr{V}_{a_{i}}, \quad \mathscr{X}_{j^{\prime}} \times_{X} \operatorname{Spec}\left(\mathcal{O}_{X, \bar{x}}\right) \simeq \mathscr{V}_{a_{i}^{\prime}} .
$$


Note also that we have a commutative diagram with exact rows

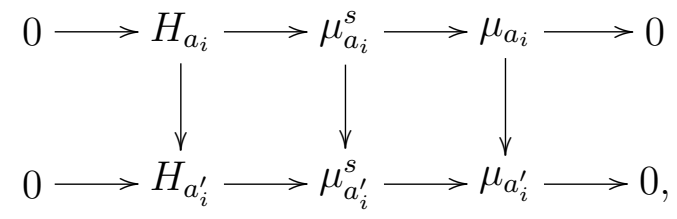

which implies that the natural isomorphism

$$
\mu_{f_{i}}^{s} \simeq \operatorname{Ker}\left(\mu_{a_{i}}^{s} \rightarrow \mu_{a_{i}^{\prime}}^{s}\right)
$$

induces an isomorphism

$$
H_{f_{i}} \simeq \operatorname{Ker}\left(H_{a_{i}} \rightarrow H_{a_{i}^{\prime}}\right) .
$$

This isomorphism identifies $K_{\bar{x}}$ with $H_{f_{i}}$, so we need to show that $H_{f_{i}}$ acts trivially on $P_{\bar{x}}$.

10.7. For any $1 \leq p, q \leq s$ there is an inclusion

$$
\sigma_{p q}: \mu_{f_{i}} \hookrightarrow H_{f_{i}}
$$

induced by the inclusion $\mu_{f_{i}} \hookrightarrow \mu_{f_{i}}^{s}$ sending $\zeta$ to the element $\left(\alpha_{k}\right) \in \mu_{f_{i}}^{s}$ with $\alpha_{p}=\zeta, \alpha_{q}=\zeta^{-1}$, and $\alpha_{k}=1$ for $k \neq p, q$.

Note that the images of the $\sigma_{p q}$ generate $H_{f_{i}}$. In fact the map

$$
\sigma_{12} \times \sigma_{23} \cdots \times \sigma_{(s-1) s}: \mu_{f_{i}} \times \cdots \times \mu_{f_{i}} \rightarrow H_{f_{i}}
$$

is an isomorphism.

Write

$$
\mathscr{V}_{a_{i}}=\left[\operatorname{Spec}\left(k\left[z_{1}, \ldots, z_{n}\right] /\left(z_{1} \cdots z_{s}\right)\right) / H_{a_{i}}\right]
$$

and let

$$
P_{a_{i}} \rightarrow \operatorname{Spec}\left(k\left[z_{1}, \ldots, z_{n}\right] /\left(z_{1} \cdots z_{s}\right)\right)
$$

be the restriction of $P$, which comes equipped with an action of $H_{a_{i}}$ lifting the action on $\operatorname{Spec}\left(k\left[z_{1}, \ldots, z_{n}\right] /\left(z_{1} \cdots z_{s}\right)\right)$.

For $1 \leq p, q \leq s$ let $T_{p q} \subset \operatorname{Spec}\left(k\left[z_{1}, \ldots, z_{n}\right] /\left(z_{1} \cdots z_{s}\right)\right)$ be the closed subscheme defined by $z_{p}=z_{q}=0$. Note that the action of the subgroup scheme $\sigma_{p q}\left(\mu_{a_{i}}\right) \subset H_{a_{i}}$ on $T_{p q}$ is trivial, therefore the restriction $\left.P\right|_{T_{p q}}$ induces a conjugacy class of homomorphisms $\sigma_{p q}\left(\mu_{a_{i}}\right) \rightarrow G$. This conjugacy class of homomorphisms has a well-defined kernel $\Sigma_{p q} \subset \sigma_{p q}\left(\mu_{a_{i}}\right)$, which is a diagonalizable group scheme being the kernel of a morphism from a diagonalizable group to a tame group scheme [AVO, 2.7]. The restriction of $\Sigma_{p q}$ to the generic point of $T_{p q}$ contains $\sigma_{p q}\left(\mu_{f_{i}}\right)$ by construction, and therefore $\Sigma_{p q}$ contains $\sigma_{p q}\left(\mu_{f_{i}}\right)$ over all of $T_{p q}$. This implies that $\sigma_{p q}\left(\mu_{f_{i}}\right)$ acts trivially on $P_{\bar{x}}$, and therefore $H_{f_{i}}$ also acts trivially on $P_{\bar{x}}$.

10.8. For general $S$, note first that the subextension $N_{S}^{\prime} \hookrightarrow N_{S}$ is determined by the induced map of constructible sheaves $\bar{N}_{S}^{\prime} \hookrightarrow \bar{N}_{S}$, and hence the preceding case of a separably closed field implies that the factorization 10.1 .1 and the descended stable torsor $P^{\prime} \rightarrow \mathscr{X}_{j^{\prime}}$ is unique if it exists. It therefore suffices to construct it étale locally on $S$. 
10.9. Let $\bar{s} \rightarrow S$ be a geometric point and fix a commutative diagram

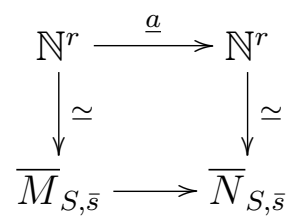

for some integers $a_{1}, \ldots, a_{r}$. The case of a separably closed field then defines a sequence of integers $a_{1}^{\prime}, \ldots, a_{r}^{\prime}$ such that $a_{i}^{\prime} \mid a_{i}$ for all $i$. As above we write $f_{i}:=a_{i} / a_{i}^{\prime}$. Let $N_{S, \bar{s}}^{\prime} \subset N_{S, \bar{s}}$ be the fiber product of the diagram

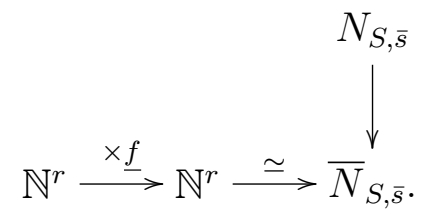

We then have a diagram of simple extensions

$$
M_{S, \bar{s}} \hookrightarrow N_{S, \bar{s}}^{\prime} \hookrightarrow N_{S, \bar{s}}
$$

By a standard limit argument we can after replacing $S$ by an étale neighborhood extend this to a diagram of simple extensions of log structures

$$
M_{S} \stackrel{j^{\prime}}{\longrightarrow} N_{S}^{\prime} \longleftrightarrow N_{S}
$$

Consider the resulting map $\mathscr{X}_{j} \rightarrow \mathscr{X}_{j^{\prime}}$. By 6.7 and 6.8 there exists an open substack $\mathscr{U} \subset \mathscr{X}_{j}$ containing the fiber over $\bar{s}$ such that $\left.P\right|_{\mathscr{U}}$ descends to the image of $\mathscr{U}$ in $\mathscr{X}_{j^{\prime}}$. Let $\mathscr{Z} \subset \mathscr{X}_{j}$ be the complement of $\mathscr{U}$ Replacing $S$ by the complement of the image of $\mathscr{Z}$ (which is closed since $\mathscr{X}_{j}$ is proper over $S$ ) we obtain an étale neighborhood of $\bar{s}$ such that $P$ descends to a torsor $P^{\prime} \rightarrow \mathscr{X}_{j^{\prime}}$. Now by construction the fiber over $\bar{s}$ of $P^{\prime}$ is stable, so by 8.7 there exists an étale neighborhood of $\bar{s}$ over which $P^{\prime}$ is stable.

\section{VERIFICATION OF THE VALUATIVE CRITERION FOR PROPERNESS}

We conclude the proof of 1.5 (i) by verifying the valuative criterion for properness for ${ }_{G} \mathscr{K}_{X / S}$.

11.1. Let $S=\operatorname{Spec}(V)$ be the spectrum of a discrete valuation ring $V$ with field of fractions $K$ and residue field $k$. We write $\eta$ (resp. $s$ ) for the generic (resp. closed) point of $S$. Fix a uniformizer $\pi \in V$.

Assume given an object

$$
\left(j_{\eta}: M_{V, \eta} \hookrightarrow N_{\eta}, P_{\eta} \rightarrow \mathscr{X}_{j_{\eta}}\right) \in_{G} \mathscr{K}_{X / S}(\operatorname{Spec}(K)) .
$$

We must show that this object extends to an object of ${ }_{G} \mathscr{K}_{X / S}(\operatorname{Spec}(V))$, after possibly replacing $V$ by a finite extension.

11.2. After possibly making an extension of $V$, we may assume that $\bar{M}_{V, s}$ is constant (note that $\bar{M}_{V, s}$ is a locally constant sheaf on $s_{\text {et }}$ ). Choose an isomorphism

$$
\mathbb{N}^{I} \oplus \mathbb{N}^{J} \simeq \bar{M}_{V}
$$


such that the induced map

$$
\mathbb{N}^{I} \rightarrow \bar{M}_{V, \bar{\eta}}
$$

is an isomorphism. We then have a canonical bijection between $J$ (resp. $I$ ) and the connected components of the singular locus which do not meet (resp. meet) the generic fiber. Fix a lifting

$$
\beta: \mathbb{N}^{I} \oplus \mathbb{N}^{J} \rightarrow M_{V}
$$

of the isomorphism 11.2.1. Then there exists a unique set of natural numbers $\left(e_{i}\right)_{i \in I}$ and isomorphism $\bar{\gamma}$ such that the following diagram commutes

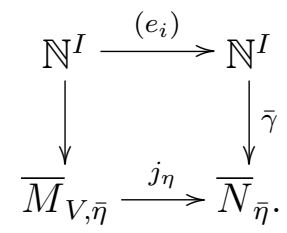

After possibly replacing $V$ by a finite extension we may assume that this diagram can be lifted to a diagram

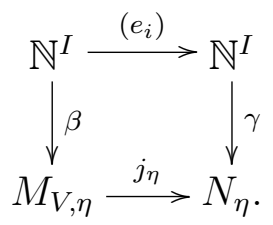

Note that the map $\gamma$ is a chart. Let $f_{i} \in V$ be the image of the $i$-th standard generator of the composite

$$
\mathbb{N}^{I} \stackrel{\beta}{\longrightarrow} \Gamma\left(\operatorname{Spec}(V), M_{V}\right) \longrightarrow V
$$

and let $g_{i} \in K$ be the image of the $i$-th standard generator under the composite

$$
\mathbb{N}^{I} \stackrel{\gamma}{\longrightarrow} \Gamma\left(\operatorname{Spec}(K), N_{\eta}\right) \longrightarrow K .
$$

Then by construction we have $g_{i}^{e_{i}} \in V$ which implies that in fact $g_{i} \in V$. Let $N^{(0)}$ be the $\log$ structure on $\operatorname{Spec}(V)$ associated to the chart

$$
\mathbb{N}^{I} \oplus \mathbb{N}^{J} \rightarrow V
$$

which restricts to $\beta$ on $\mathbb{N}^{J}$ and sends the $i$-th standard generator of $\mathbb{N}^{I}$ to $g_{i}$. We then have a simple extension

$$
j^{(0)}: M_{V} \hookrightarrow N^{(0)}
$$

over $\operatorname{Spec}(V)$ restricting to $j_{\eta}$ over the generic point.

We therefore get a stack

$$
\mathscr{X}_{j(0)} \rightarrow \operatorname{Spec}(V)
$$

with a $G$-torsor

$$
P_{\eta} \rightarrow \mathscr{X}_{j^{(0)}, \eta} \cdot
$$

11.3. Let $\mathscr{Z} \subset \mathscr{X}_{j^{(0)}}$ be the union of the connected components of the singular locus of $\mathscr{X}_{j^{(0)}}$ which are contained in the closed fiber, and let $Z \subset X$ denote the image in $X$. Then by 5.1 and $4.3 P_{\eta}$ extends to a $G$-torsor

$$
P^{\prime} \rightarrow \mathscr{X}_{j^{(0)}}^{\prime}:=\mathscr{X}_{j^{(0)}} \backslash \mathscr{Z} .
$$


11.4. For each $j \in J$ write the image of the $j$-th standard generator under the map

$$
\mathbb{N}^{J} \longrightarrow \Gamma\left(V, M_{V}\right) \longrightarrow V
$$

as $u_{j} \pi^{k_{j}}$ where $u_{j} \in V^{*}$ and $k_{j} \in \mathbb{N}$. Let $N$ be the $\log$ structure on $\operatorname{Spec}(V)$ induced by the map

$$
\mathbb{N}^{I} \oplus \mathbb{N}^{J} \rightarrow V
$$

whose restriction to $\mathbb{N}^{I}$ is the map defined by $N^{(0)}$ and which sends the $j$-th standard generator of $\mathbb{N}^{J}$ to $\pi$. The map

$$
\mathbb{N}^{I} \oplus \mathbb{N}^{J} \rightarrow V^{*} \oplus \mathbb{N}^{I} \oplus \mathbb{N}^{J}, \quad e_{i} \mapsto e_{i} \quad(i \in I), \quad e_{j} \mapsto\left(u_{j}, 0, k_{j} e_{j}\right) \quad(j \in J)
$$

induces a morphism of $\log$ structures $N^{(0)} \rightarrow N$. Let $j: M_{V} \rightarrow N$ be the composite

$$
M_{V} \rightarrow N^{(0)} \rightarrow N
$$

We then obtain a morphism of stacks

$$
\mathscr{X}_{j} \rightarrow \mathscr{X}_{j(0)}
$$

which is an isomorphism away from $\mathscr{Z}$. In particular, the torsor $P^{\prime}$ can be viewed as a torsor over

$$
\mathscr{X}_{j}^{\prime}:=\mathscr{X}_{j} \times_{X}(X \backslash Z) .
$$

Now observe that for any geometric point $\bar{z} \rightarrow Z$ there exists an étale neighborhood $U$ of $\bar{z}$ in $X$ such that

$$
\mathscr{X}_{j} \times_{X} U \simeq\left[\operatorname{Spec}\left(V\left[x_{1}, \ldots, x_{n}\right] /\left(x_{1} \cdots x_{s}-\pi\right)\right) / \Delta\right]
$$

for some $s \leq n$ (here $\Delta$ and the action is as in 3.3). It follows that $P^{\prime}$ extends to a torsor $P \rightarrow \mathscr{X}_{j}$ (by 3.7).

11.5. We therefore obtain a twisted $G$-torsor $\left(j: M_{V} \hookrightarrow N, P\right)$ over $X$ whose restriction to $X_{\eta}$ is our given object of ${ }_{G} \mathscr{K}_{X / S}(\operatorname{Spec}(K))$. Applying the stabilization construction of section 10 we then obtain the desired object of ${ }_{G} \mathscr{K}_{X / S}(\operatorname{Spec}(V))$.

This completes the proof of 1.5 (i).

\section{THE STACK ${ }_{G} \mathscr{K}_{X / S}$ IS TAME.}

12.1. Let $\left(X, M_{X}\right) /\left(S, M_{S}\right)$ be as in 7.1 , and let $\left(j: M_{S} \rightarrow N_{S}, P\right)$ be an object of ${ }_{G} \mathscr{K}_{X / S}(S)$. It suffices to show that the geometric fibers of the automorphism group scheme of $(j, P)$ is tame. So we assume that $S=\operatorname{Spec}(k)$ is the spectrum of an algebraically closed field, and let $\mathscr{A}$ denote the automorphism group scheme of $(j, P)$. The group scheme $\mathscr{B}$ of automorphisms of $N_{S}$ which restrict to the identity on $M_{S}$ is isomorphic to a product of group schemes of the form $\mu_{a}$, and there is a natural homomorphism

$$
\mathscr{A} \rightarrow \mathscr{B} \text {. }
$$

Let $K \subset \mathscr{A}$ be the kernel of this homomorphism. Since $\mathscr{B}$ is diagonalizable, to prove that $\mathscr{A}$ is tame it suffices to show that $K$ is tame. 
12.2. The group scheme $K$ is the group scheme of automorphisms of the $G$-torsor $P$ over $\mathscr{X}_{j}$. Write $G$ as an extension

$$
1 \rightarrow \Delta \rightarrow G \rightarrow H \rightarrow 1
$$

where $\Delta$ is diagonalizable and $H$ is tame and étale. Let $P_{H}$ denote the quotient $P / \Delta$, which is an $H$-torsor over $\mathscr{X}_{j}$. The automorphism group scheme of $P_{H}$ over $\mathscr{X}_{j}$ is a twisted form $\mathscr{H}$ of $H$. Let $\mathscr{C}$ denote the tame étale group scheme over $\operatorname{Spec}(k)$ corresponding to the group $\Gamma\left(\mathscr{X}_{j}, \mathscr{H}\right)$. By the proper base change theorem, $\mathscr{C}$ represents the functor which to any $k$-scheme $T$ associates the group of automorphisms of the base change of $P_{H}$ to $T$. We therefore have a natural homomorphism

$$
K \rightarrow \mathscr{C}
$$

whose kernel $K^{\prime}$ is the subgroup scheme of automorphisms of $P$ over $P_{H}$. To prove that $K$ is tame it then suffices to show that $K^{\prime}$ is tame, and for this in turn it suffices to show that the group scheme of automorphisms of $P$ over $P_{H}$ is tame.

12.3. Let $Z / k$ denote $\operatorname{Spec}\left(\Gamma\left(P_{H}, \mathscr{O}_{P_{H}}\right)\right)$. Since $P_{H}$ is reduced the scheme $Z$ is equal to a finite disjoint union of copies of $\operatorname{Spec}(k)$. Since $\Delta$ is abelian the group scheme of automorphisms of $P$ over $P_{H}$ is canonically isomorphic to the scheme

$$
\underline{\operatorname{Hom}}_{k}(Z, \Delta) \text {, }
$$

which is isomorphic to a finite product of copies of $\Delta$. In particular, this is a tame group scheme.

This completes the proof of 1.5 (ii).

\section{REFERENCES}

[AV] D. Abramovich and A. Vistoli, Compactifying the space of stable maps, J. Amer. Math. Soc 15 (2002), $27-75$.

[AVO] D. Abramovich, M. Olsson, and A. Vistoli, Tame stacks in positive characteristic, to appear in Anneles de l'Institut Fourier.

[AVO2] _ Twisted stable maps to tame Artin stacks, preprint (2008).

[Ar] M. Artin, Algebraization of formal moduli. I, in Global Analysis (Papers in Honor of K. Kodaira) (1969), 21-71, Univ. Tokyo Press, Tokyo.

[Ar2] _ Algebraic approximation of structures over complete local rings, Publications Mathmatiques de l'IHS 36 (1969), 23-58.

[SGA4] M. Artin, A. Grothendieck, and J.-L. Verdier, Théorie des topos et cohomologie étale des schémas., Lecture Notes in Mathematics 269, 270, 305, Springer-Verlag, Berlin (1972).

[DM] P. Deligne and D. Mumford, The irreducibility of the space of curves of given genus, Inst. Hautes tudes Sci. Publ. Math. 36 (1969), 75-109.

[SGA1] A. Grothendieck, Revêtements étales et groupe fondamental, Lectures Notes in Math 224 (1971).

[Ka] K. Kato, Logarithmic structures of Fontaine-Illusie, Algebraic analysis, geometry, and number theory (Baltimore, MD, 1988), Johns Hopkins Univ. Press, Baltimore, MD, 1989, 191-224.

[MO] K. Matsuki and M. Olsson, Kawamata-Viehweg vanishing as Kodaira vanishing for stacks, Math. Res. Letters 12 (2005), 207-217.

[Ol] M. Olsson, Universal log structures on semi-stable varieties, Tohoku Math. J. 55 (2003), 397-438.

[Ol2] _ On (log) twisted curves, Comp. Math. 143 (2007), 476-494.

[Ol3] _ On proper coverings of Artin stacks, Adv. Math. 198 (2005), 93-106.

[Ol4] L Logarithmic geometry and algebraic stacks, Ann. Sci. d'ENS 36 (2003), 747-791.

[Og] A. Ogus, Lectures on logarithmic algebraic geometry, book in preparation (2008). 